\title{
Heads, Shoulders, Elbows, Knees, and Toes: Modular Gdf5 Enhancers Control Different Joints in the Vertebrate Skeleton
}

\author{
Hao Chen ${ }^{1 \odot}$, Terence D. Capellini ${ }^{1,2,3}$, Michael Schoor ${ }^{4}$, Doug P. Mortlock ${ }^{5}$ A. \\ Hari Reddi ${ }^{6}$, David M. Kingsley ${ }^{1,7 *}$
}

1 Department of Developmental Biology, Beckman Center B300, Stanford University School of Medicine, Stanford, California, United States of America, 2 Human Evolutionary Biology, Peabody Museum, Harvard University, Cambridge, Massachusetts, United States of America, 3 Broad Institute of MIT and Harvard, Cambridge, Massachusetts, United States of America, 4 Miltenyi Biotec GmbH, Bergisch Gladbach, Germany, 5 Molecular Physiology and Biophysics and Vanderbilt Genetics Institute, Vanderbilt University, Nashville, Tennessee, United States of America, 6 Center for Tissue Regeneration and Repair, University of California Davis Medical Center, Sacramento, California, United States of America, 7 Howard Hughes Medical Institute, Stanford University, Stanford, California, United States of America

- These authors contributed equally to this work.

* kingsley@stanford.edu

\section{f openaccess}

Citation: Chen H, Capellini TD, Schoor M, Mortlock DP, Reddi AH, Kingsley DM (2016) Heads, Shoulders, Elbows, Knees, and Toes: Modular Gdf5 Enhancers Control Different Joints in the Vertebrate Skeleton. PLoS Genet 12(11): e1006454. doi:10.1371/journal.pgen.1006454

Editor: Fanxin Long, WUSTL, UNITED STATES

Received: July 31, 2016

Accepted: November 2, 2016

Published: November 30, 2016

Copyright: ๑ 2016 Chen et al. This is an open access article distributed under the terms of the Creative Commons Attribution License, which permits unrestricted use, distribution, and reproduction in any medium, provided the original author and source are credited.

Data Availability Statement: All relevant data are within the paper and its Supporting Information files.

Funding: This work was supported in part by a German Research fellowship (Michael Schoor), two Arthritis Foundation Postdoctoral Fellowships (Hao Chen and Michael Schoor), and by National Institutes of Health Grant AR42236 (David M. Kingsley). David M. Kingsley is an investigator of the Howard Hughes Medical Institute. The funders had no role in study design, data collection and analysis, decision to publish, or preparation of the manuscript.

\section{Abstract}

Synovial joints are crucial for support and locomotion in vertebrates, and are the frequent site of serious skeletal defects and degenerative diseases in humans. Growth and differentiation factor 5 (Gdf5) is one of the earliest markers of joint formation, is required for normal joint development in both mice and humans, and has been genetically linked to risk of common osteoarthritis in Eurasian populations. Here, we systematically survey the mouse Gdf5 gene for regulatory elements controlling expression in synovial joints. We identify separate regions of the locus that control expression in axial tissues, in proximal versus distal joints in the limbs, and in remarkably specific sub-sets of composite joints like the elbow. Predicted transcription factor binding sites within Gdf5 regulatory enhancers are required for expression in particular joints. The multiple enhancers that control Gdf5 expression in different joints are distributed over a hundred kilobases of DNA, including regions both upstream and downstream of Gdf5 coding exons. Functional rescue tests in mice confirm that the large flanking regions are required to restore normal joint formation and patterning. Orthologs of these enhancers are located throughout the large genomic region previously associated with common osteoarthritis risk in humans. The large array of modular enhancers for Gdf5 provide a new foundation for studying the spatial specificity of joint patterning in vertebrates, as well as new candidates for regulatory regions that may also influence osteoarthritis risk in human populations. 
Competing Interests: The authors have declared that no competing interests exist.

\section{Author Summary}

Joints, such as the hip and knee, are crucial for support and locomotion in animals, and are the frequent sites of serious human diseases such as arthritis. The Growth and differentiation factor 5 (Gdf5) gene is required for normal joint formation, and has been linked to risk of common arthritis in Eurasians. Here, we surveyed the mouse gene for the regulatory information that controls Gdf5's expression pattern in stripes at sites of joint formation. The gene does not have a single regulatory sequence that drives expression in all joints. Instead, Gdf5 has multiple different control sequences that show striking specificity for joints in the head, vertebral column, shoulder, elbow, wrist, hip, knee, and digits. Rescue experiments show that multiple control sequences are required to restore normal joint formation in $G d f 5$ mutants. The joint control sequences originally found in mice are also present in humans, where they are marked as active regions during fetal development and post-natal life, and map to a large region associated with arthritis risk in human populations. Regulatory variants in the human GDF5 control sequences can now be studied for their potential role in altering joint development or disease risk at particular locations in the skeleton.

\section{Introduction}

Synovial joints are the key articulations that connect bones and allow movement in vertebrate skeletons. A typical synovial joint consists of the ends of two long bones, thin layers of articular cartilage covering the bones, a joint cavity filled with synovial fluid, and ligaments and a fibrous capsule that connect the bones and surround the joint. The articular cartilage helps distribute load and provides a smooth lubricated surface to facilitate motion, while the connecting ligaments and capsule provide tethered, guided movement across the joint [1].

Disorders of joint formation and maintenance, including congenital malformations, physical trauma, and degenerative diseases, effect hundreds of millions of people around the world. Most notably, osteoarthritis (OA) in which the articular cartilage and subjacent bone degrade causing substantial joint pain and loss of mobility, affects $13.9 \%$ of adults aged 25 and older, and 33.6\% of adults aged 65 and older [2]. Despite the prevalence of synovial joint disorders in public health, little is known about the genetic and molecular mechanisms that control joint formation and maintenance, or that control the differential sensitivity of specific joints to risk of OA $[3,4]$.

Bone morphogenetic proteins (BMPs) are key signaling molecules and receptors known to regulate various aspects of skeletal development $[5,6]$. Growth and differentiation factor 5 (Gdf5) was originally isolated as a novel member of this family [7, 8]. Subsequent studies have shown that $G d f 5$ is one the earliest known markers of joint formation [9-12]. It is initially expressed in a dramatic pattern of stripes that form 24-36 hours before the histological appearance of the interzone, a region of prechondrogenic mesenchyme fated to become the joint cavity. Subsequently, Gdf5 expressing cells contribute to many, if not all, adult soft and hard tissue joint structures, including articular cartilage, joint capsule, and ligaments [13, 14].

Genetic studies show that $G d f 5$ has conserved roles in normal formation and maintenance of synovial joints in both mice and humans, as well as control of long bone growth. The classic recessive brachypodism ( $b p$ ) [15] mutation consists of frameshift changes in the mouse Gdf5 gene [7]. This viable mutation causes mild reduction of long bone lengths and severe shortening of paws, the latter due to both shorter metapodial bones and loss of one of the three phalanges found in digits rays II-V [16]. Brachypodism mutant mice also lack particular synovial 
joints in the digits, wrists, and ankles [7, 15], form knee joints without the anterior and posterior cruciate ligaments, and develop OA when experimentally challenged [17, 18]. Although brachypodism mutants have defects in only a subset of the joints and skeletal structures where Gdf5 is normally expressed, additional joint and growth defects are revealed in double mutants with other BMP family members, confirming that $G d f 5$ also functions in formation of many joints in the limb, sternum, and vertebral column, and in control of multiple skeletal structures $[9,19]$.

Loss of function mutations in the human GDF5 gene are also found in patients with acromesomelic chondrodysplasia Hunter-Thompson syndrome [20, 21], Grebe syndrome [22], Brachydactyly Type A2 [23] and C [24, 25]. Some human mutations cause brachypodism-like defects at birth, such as the reduction in phalangeal number and the loss of synovial joints. In other syndromes, such as Angel-shaped phalango-epiphyseal dysplasia, hip joint malformations are often coupled with OA [26].

Recent genome-wide and candidate gene association studies show that the genomic interval surrounding GDF5 can alter risk of common adult OA in humans, even in the absence of obvi-

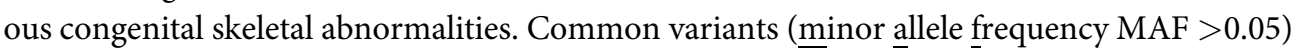
spanning a large $130 \mathrm{~kb}$ interval from GDF5 through the downstream gene (Ubiquinol-Cytochrome C Reductase Complex Chaperone-UQCC) are significantly associated with a 2-fold increase in hip and knee OA risk $[27,28]$. The most studied risk alleles, present in high frequency in Eurasians, are located in the 5' untranslated region (UTR) of GDF5 [27]. These variants, when tested in constructs containing a GDF5 minimal promoter region, have been shown to reduce transcriptional activity in articular chondrocyte cells, and are expressed at lower levels than alternate alleles in joint cartilages from total knee replacement patients [27, $29,30]$. No common coding region variants have been identified in previous association studies or in deep exome sequencing projects on OA patients that can explain GDF5 population level associations with increased OA risk [31-33]. Thus, additional causal regulatory variants may remain undiscovered in the region.

Here, we systematically survey the mouse $G d f 5$ locus to map the regulatory sequences controlling expression and function. We identify a large array of non-coding regions that control expression in different subsets of developing joints. Many of the enhancers show surprising specificity for different subsets of joints, due in part to predicted transcription factor binding sites that we show are required for spatial specificity. We find that full functional rescue of mouse phenotypes depends on both upstream and downstream regulatory regions. Strikingly, orthologous regions are found throughout the $130 \mathrm{~kb}$ region previously associated with $\mathrm{OA}$, identifying new candidate regions for future functional studies of disease risk in humans.

\section{Results}

\section{A BAC scan of the Gdf5 locus reveals upstream and downstream regulatory domains}

To identify cis-regulatory elements precisely controlling Gdf5 expression in joints, we initially performed a BAC scan encompassing a $250 \mathrm{~kb}$ genomic interval centered on the mouse Gdf5 locus. We first chose a BAC that covers $110 \mathrm{~kb}$ upstream to $30 \mathrm{~kb}$ downstream of the Gdf5 coding region and modified it by inserting an Internal Ribosome Entry Site (IRES)$\beta G e o$ reporter cassette into the gene's 3'UTR ("Upstream BAC", Fig 1A). The IRES reporter cassette allows dicistronic translation of both the GDF5 and LacZ proteins from Gdf5 mRNA. We then tested this construct's ability to drive lacZ expression in multiple independent transgenic mouse embryos at embryonic day 14.5 (E14.5), a key period when synovial joints form. The Upstream BAC drove consistent expression in axial and appendicular sites 


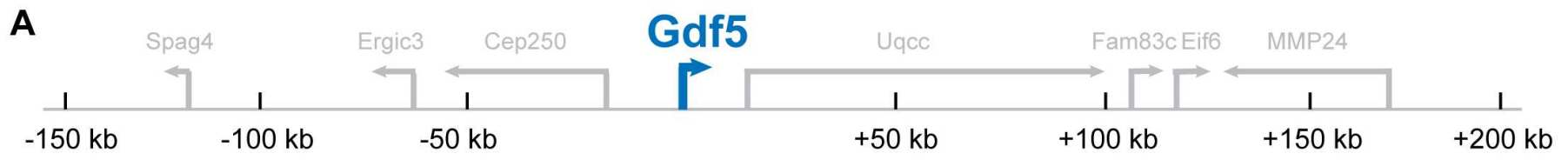

\section{Upstream BAC}

\section{Downstream BAC}

B

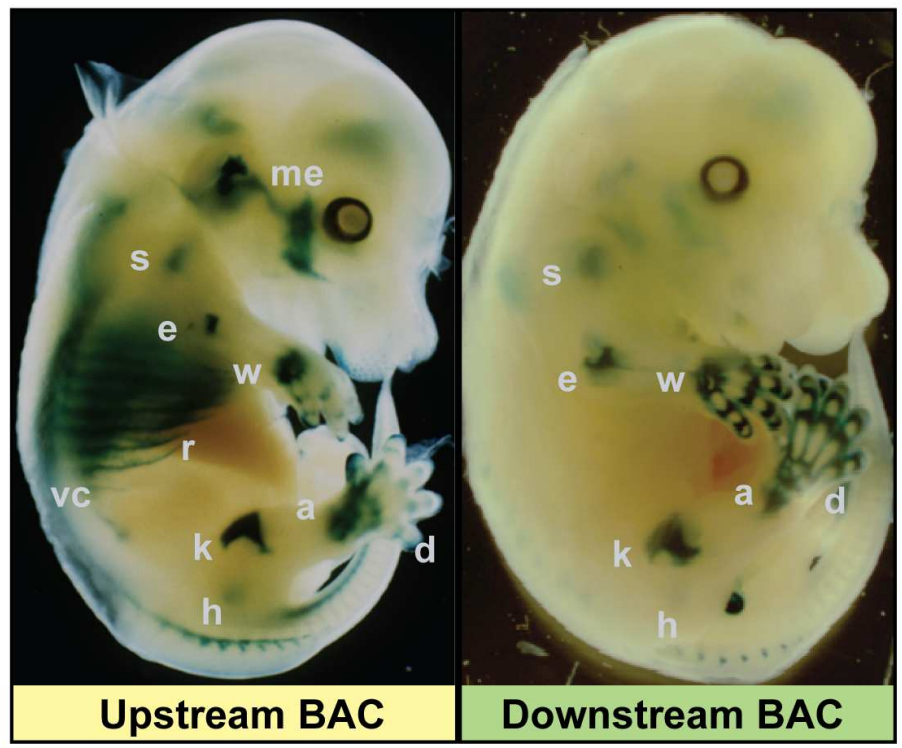

C

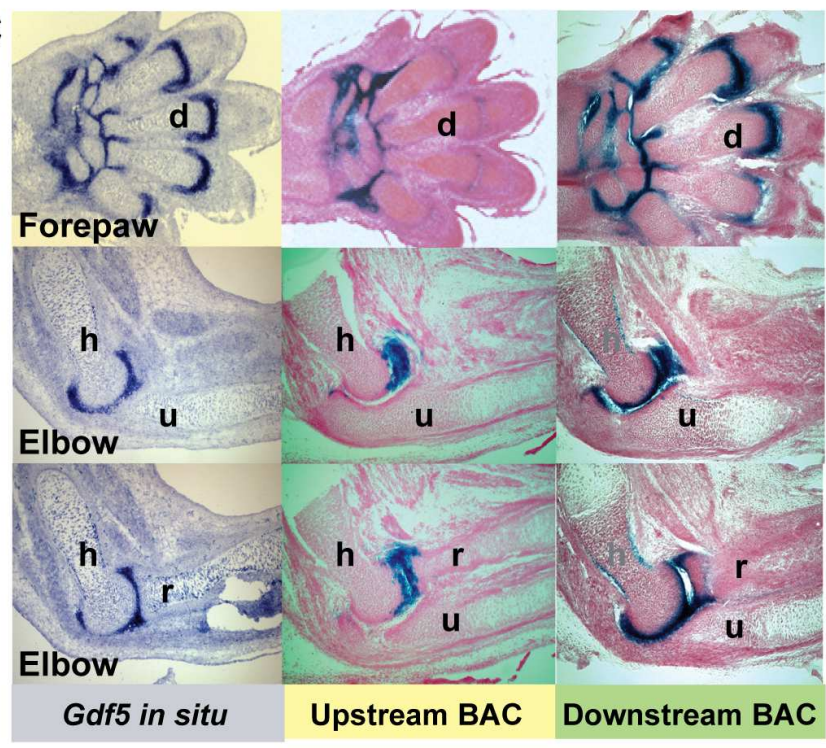

Fig 1. BAC scan of Gdf5 region reveals both upstream and downstream regulatory domains for joint expression. (A) Relative location of two BACs spanning the Gdf5 region. Upstream BAC (yellow) extends $110 \mathrm{~kb}$ upstream to $30 \mathrm{~kb}$ downstream of Gdf5 coding exons, while the Downstream BAC (green) spans an additional $109 \mathrm{~kb}$ downstream. IRES- $\beta$ Geo cassette insertion (blue bar) within the 3'UTR of the Gdf5 coding exons of each BAC generates a visual reporter of Gdf5 expression. (B) Representative transgenic embryos at E14.5 derived from each BAC. Both BACs drive lacZ expression in synovial joints of the proximal limb, such as the shoulder (s), elbow (e), hip (h, expression is deep in the tissue), and knee (k); as well as the distal limb, such as the wrist (w), ankle (a), and digit joints (d). Additionally, expression was detected in axial tissues such as the ribs (r), in and around vertebral column (vc), and some sites in the head, such as the middle ear (me). In general, the Upstream BAC drove stronger proximal limb expression while the Downstream BAC drove stronger distal limb expression. (C) E14.5 embryonic forepaw and elbow sections comparing the endogenous Gdf5 expression pattern via in situ hybridization (left) with the lacZ patterns driven by the Upstream (middle) and the Downstream BACs (right). Note that the endogenous Gdf5 gene is expressed in stripes at most sites of joint formation, including both the humeroulnar (h-u) joint and the humeroradial $(\mathrm{h}-\mathrm{r}$ ) joints of the elbow. The Upstream BAC drives expression in many proximal limb joints, but only in the humeroradial and not the humeroulnar joint of the elbow. In contrast, the Downstream BAC drives comprehensive appendicular joint expression, including both joints of the elbow. All expression patterns were confirmed in multiple independent transgenic embryos (S1 Table).

doi:10.1371/journal.pgen.1006454.g001

characteristic of the endogenous $G d f 5$ expression pattern (Fig 1B). In the axial skeleton, lacZ was detected in ribs, the vertebral column, and in head structures such as the middle ear and mandible (Fig 1B) among other sites (S1 Fig). Each of these sites corresponds to locations where the endogenous $G d f 5$ gene is also expressed ( 1 Fig). In the limb, lacZ expression was found in proximal synovial joints of the shoulder, elbow, hip, and knee, as well as distal joints of the wrist, ankle, and digits, albeit expression was both lower and less reproducible in these distal domains (S1 Table). Serial sections of X-gal stained limbs showed that extensive lacZ expression was present in the wrist and ankle regions, with weaker signal in the digit joints, especially compared to the endogenous Gdf5 expression (Fig 1C, top panel). In the elbow region, whereas endogenous $G d f 5$ gene expression was detected in both the humeroradial 
(h-r) and humeroulnar (h-u) joints, lac $Z$ was detected only in the humeroradial joint (h-r) (Fig 1C, middle and bottom panels). Therefore, while the Upstream BAC contains key regulatory elements that regulate $G d f 5$ expression in some joints, it appears to lack regulatory sequence that can faithfully recapitulate the complete $G d f 5$ expression pattern.

To expand our search for additional joint control elements, we isolated a second BAC that extends $\sim 110 \mathrm{~kb}$ further downstream of our first BAC, and again inserted an IRES- $\beta$ Geo reporter cassette into the same position in the Gdf5 3'UTR ("Downstream BAC", Fig 1A). In E14.5 transgenic mouse embryos, this Downstream BAC drove striking lac $Z$ expression in all proximal synovial joints of the shoulder, elbow, hip, and knee, as well as in distal joints of the wrist, ankle, and digits (Fig 1B). It additionally drove expression in the same axial and head structures as the Upstream BAC, albeit at much weaker levels, possibly because of repressor sequences also present in the Downstream BAC interval or positional effects of BAC transgene integration (Fig 1B, S1 Table). Compared to the Upstream BAC pattern, lacZ expression was much stronger in the transverse joint stripes of the digits and encompassed a wider domain that included the mesenchyme of the inter-digital space (IDS) and each digit's lateral and medial border (Fig 1C, top panel). Further inspection revealed that, unlike the Upstream BAC, the Downstream BAC drove lac $Z$ expression across both the humeroradial (h-r) and humeroulnar (h-u) joints in the elbow (Fig $1 \mathrm{C}$, middle and bottom panels). Overall, the lacZ expression pattern produced by the Downstream BAC closely recapitulated the endogenous Gdf5 expression pattern in limbs.

\section{Downstream and upstream BAC sequences continue to drive strong joint expression in adulthood}

We further assessed the regulatory control of $G d f 5$ at later stages of joint development when joint structures, such as tendons, ligaments, articular capsules, and articular cartilages mature. In metapodial-phalangeal joints, intervertebral joints, as well as the calcaneal insertion site of the Achilles tendon, the Upstream BAC continued to drive lacZ expression in adult animals (S2 Fig). In the knee at E17.5 we found that the Downstream BAC drove strong lacZ expression in nearly all joint tissues and surfaces, whereas the Upstream BAC drove weak expression in very restricted domains such as the most inferior and superior articular surfaces of the femur and tibia, respectively, as well as in the developing cruciate ligaments (S3 Fig). The strong knee expression exhibited by the Downstream BAC continued during post-natal development and through adult joint maintenance stages (S3 Fig). Conversely, Upstream BAC sequences failed to drive detectable lacZ expression via X-gal staining techniques in the knee during adulthood (S3 Fig). These data reveal that the upstream and downstream regions contain enhancers active during both early joint formation, and in joint homeostasis during postnatal life. Such expression is expected, since endogenous $G d f 5$ expression has also been detected in the adult articular cartilage and joint tissues of both mice and humans [29, 34, 35].

\section{Two separable upstream sequences of Gdf5 drive expression in axial structures and proximal synovial joints}

To further identify specific non-coding sequences controlling different anatomical patterns, we first took advantage of the finding that the expression pattern of $G d f 5$ is well conserved between humans [29], mice [9], and chicken [11]. We used VISTA and PIP maker (Methods) to identify multiple non-coding regions that were also well conserved across species (Fig 2A). We then cloned different non-coding conserved regions upstream of a minimal promoter and lac $Z$ reporter, and tested the ability of different constructs to drive specific patterns in E14.5 mouse embryos. 
A

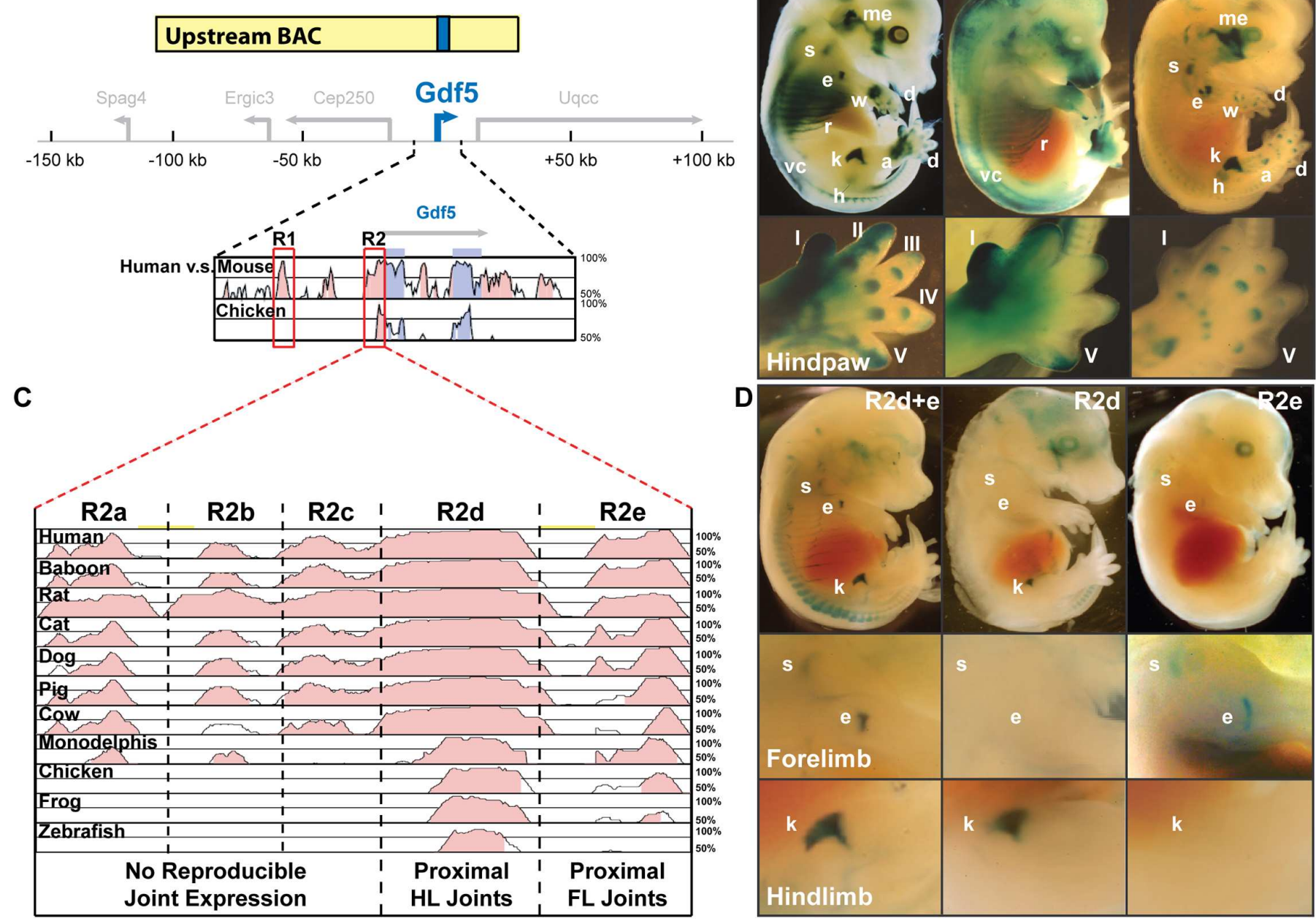

Fig 2. Two upstream regulatory elements drive expression in axial tissue domains (R1) and limb joints (R2); while separate sub-domains of R2 control distinct positions with limbs. (A) Evolutionarily conserved non-coding regions (pink peaks) were identified upstream of Gdf5 coding regions (blue peaks), based on a criterion of $70 \%$ or greater nucleotide sequence identity between human, mouse, and chicken sequence over a region of $300 \mathrm{bp}$ or more. (B) Two distinct conserved non-coding peaks (R1 and R2; red-boxes in A) were tested for enhancer activity at E14.5 and compared to lacZ expression driven by the Upstream BAC. R1 drove expression in axial tissues such as the ribs ( $\mathrm{r}$ ), vertebral column (VC), and anterior mesenchyme of the paw, while R2 drove expression in limb joints such as the shoulder (s), elbow (e), wrist (w), hip (h), knee (k), ankle (a), and digit regions (d). Bottom panel shows zoom-in images of hindpaws. (C) Comparison of genomic sequences of R2 from twelve species with mouse as reference. Based on patterns of conservation between species, R2 was divided into five sub-regions (a-e). (D) Transgenic embryos at E14.5 from subregions R2d+e (left), R2d (middle), and R2e (right). Sub-region R2d drove hindlimb (HL) joint expression, while sub-region R2e drove forelimb (FL) joint expression. Panels below show zoom-in images of forelimb (shoulder, elbow) and hindlimb (knee) from R2d+e, R2d, and R2e embryos.

doi:10.1371/journal.pgen.1006454.g002

Two regions, contained within the upstream Gdf5 domain shared by both BACs, drove relevant expression patterns (Fig 2A, red boxes). Region R1 (754 bp), located $5 \mathrm{~kb}$ upstream of Gdf5, controlled lacZ expression in many of the axial sites previously described for each BAC (Fig 2B). These domains include the ribs, the vertebral column, and sites within the head and jaw (S1 Table). This sequence also drove wide expression in the mesenchyme of the anterior interdigital space of the fore- and hindpaw (Fig 2B). Region R2 (802 bp), adjacent to the promoter region of $G d f 5$, drove consistent lac $Z$ expression in the proximal synovial joints of the shoulder, elbow, hip, and knee (Fig 2B; S1 Table). However, as observed with the Upstream $\mathrm{BAC}$, this element did not drive expression in the humeroulnar (h-u) joint (Fig $2 \mathrm{~B}$ and 
S4B Fig, bottom panels) and expression in the digital joints was weak and less reproducible (Fig 2B). Importantly, these two evolutionarily conserved non-coding elements when considered in toto drove lacZ in a pattern closely resembling the Upstream BAC (Fig 2B).

\section{Different subregions of the $\mathrm{R} 2$ upstream element drive preferential expression in forelimb or hindlimb joints}

To further delineate specific joint expressing domains within the R2 regulatory region, we used truncation mapping in the context of a comparative genomic sequence analysis.

Sequences from twelve different species (human, baboon, mouse, rat, cat, dog, pig, cow, opossum, chicken, frog, and zebrafish) were precisely aligned using Vista (Fig 2C) to identify conserved sub-regions within R2. Analysis of sequence conservation across this element revealed at least 5 sub-regions of weak to strong conservation (R2a-e). While sub-regions $\mathrm{R} 2 \mathrm{a}+\mathrm{b}+\mathrm{c}$ were weakly conserved; sub-region R2d was particularly well conserved across a diverse set of placental mammals, opossum, chicken, and zebrafish; and sub-region R2e was conserved to frog. Given these differences in conservation, sub-regions $\mathrm{R} 2 \mathrm{a}+\mathrm{b}+\mathrm{c}$ and $\mathrm{R} 2 \mathrm{~d}+\mathrm{e}$ were cloned separately into our lacZ reporter system and tested for their expression in E14.5 transgenic embryos. Sub-regions $\mathrm{R} 2 \mathrm{a}+\mathrm{b}+\mathrm{c}$ did not drive any reproducible pattern of lacZ expression (S1 Table). On the other hand, sub-regions $\mathrm{R} 2 \mathrm{~d}+\mathrm{e}$ together drove lac $Z$ expression in the proximal limb joints of the shoulder, elbow, hip, and knee, but not in distal digit joints (Fig 2D). Interestingly, in the elbow joint, sub-region $\mathrm{R} 2 \mathrm{~d}+\mathrm{e}$ drove $l a c Z$ in the humeroradial articulation only (Fig 2D), a restricted pattern identical to that observed for the complete R2 element (Fig 2B and S4B Fig) and the Upstream BAC (Fig 1B and 1C).

To further examine the regulatory capacity of sub-regions R2d versus R2e, smaller constructs containing each (i.e., R2d or R2e) were individually tested in our lacZ reporter assay in transgenic embryos. Sub-region R2d drove lac $Z$ expression only in the proximal joints of the hindlimb (i.e. hip and knee) (Fig 2D, R2d). In contrast, sub-region R2e regulated lacZ expression only in the proximal joints of the forelimb (i.e. shoulder and elbow) (Fig 2D, R2e). These data provide striking evidence that expression in particular fore- and hindlimb joints can be regulated by separate DNA elements.

\section{Predicted transcription factor binding sites are required for expression in particular joints}

To look for possible factors that may contribute to joint-specific patterns, we used two different programs to identify putative transcription factor binding sites in the R2 element, the UNIPROBE database [36] and rVista/MatInspector [37-40]. At the recommended enrichment of 0.4, UNIPROBE predicted binding sites for a number of different transcription factors (S2 Table), of which, many are homeodomain proteins that overlap in their binding site preferences. One of these potential upstream factors, BARX, is a homeodomain protein with known roles in chondrogenesis [41]. In situ hybridization studies in mouse and chick reveal that BARX1 and BARX2 family members remarkably overlap in expression with $G d f 5$ in proximal and distal joints [41-44]. Given the known roles of BARX2 in chondrogenesis in the mouse, we used the BARX motif to further search for conserved homeodomain binding sites between humans and mouse. We identified 8 predicted BARX2 sites (S1-S8) in the mouse R2 region at a UNIPROBE enrichment cutoff of 0.4. Four of these predicted sites are mouse-specific (S1, S3, S4, and S7), and four are additionally conserved using the same UNIPROBE enrichment cutoff in the human R2 ortholog (S2, S5, S6, and S8) (Fig 3A; Methods). Note that because of overlap of the binding specificities of many homeodomain containing factors, these sites could also represent binding sites for other factors besides BARX proteins. 
A
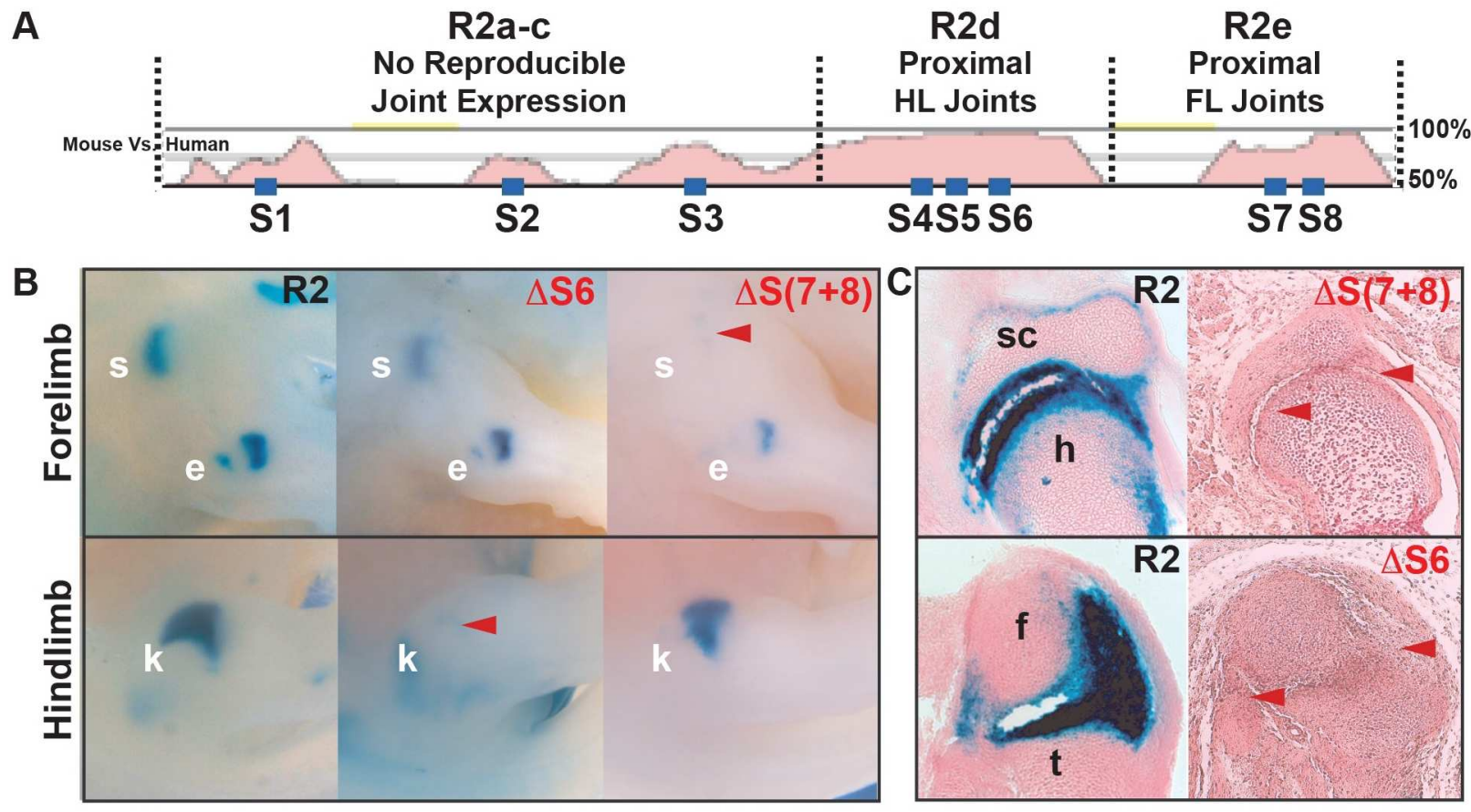

Fig 3. Predicted homeodomain binding sites are required for R2 expression domains in specific joints. (A) Eight predicted homeodomain binding sites (S1-S8, blue boxes) are found across R2 subregions. Four of these UNIPROBE predicted sites are found in both mice and humans, including S2 in sub-region R2a-c, S5 and S6 in sub-region R2d, and S8 in sub-region R2e. (B) Targeted mutagenesis of conserved S6 within the hindlimb regulatory zone of R2 eliminates normal lacZsignal in the knee (red arrowhead) but not elbow or shoulder, tissues where this enhancer region is predicted to have little to no influence on expression. Mutagenesis of sites $S(7+8)$ within the forelimb regulatory zone of R2 eliminates expression in shoulder (red arrowhead) but not elbow or knee, tissues where this sequence is predicted to have little to no influence on expression. (C) Histology at E14.5 of wild-type R2 (left) versus mutant $\mathrm{R} 2$ (right) constructs reveals specific reductions (red arrowheads) in joint domains for each construct. Abbreviations: s, shoulder; e, elbow; k, knee; sc, scapula; $h$, humerus; f, femur; t, tibia.

doi:10.1371/journal.pgen.1006454.g003

We next wanted to test in vivo whether any of the predicted homeodomain (e.g., BARX2) binding sites are required for lacZ expression. Since sites S1-S3 lie in a R2 sub-region that yielded no reproducible enhancer activity, we concentrated on sites S4-S6 in the hindlimb (hip/knee) sub-region, and on S7-S8 in the forelimb (shoulder/elbow) sub-region. Using a database of experimentally measured binding interactions between many vertebrate transcription factors and all possible 8-mer target sequences (UNIPROBE, Methods), we found that changing 3 or 4 bases in the predicted BARX2 site was sufficient to reduce experimentally determined BARX2 binding to values below significance $(\mathrm{P}>0.05)$ and enrichment $(\mathrm{E}<0.4)$ thresholds (S3 Table, Methods). We therefore generated new constructs containing the R2 enhancer with different predicted BARX2 sites modified, and tested for effects on expression patterns in transgenic mouse embryos at E14.5. While mutation of individual sites S4 or S5 had no observable effects, mutation of S6 eliminated expression within the knee (Fig 3B), but not the hip (S1 Table) in multiple transgenic lines tested. Histological analysis revealed that lac $Z$ expression was absent from the capsule and articular joint surfaces in the knee. Mutation of S7 had no visible effect, while mutation of S8 slightly reduced scapulo-humeral expression (S1 Table). However, mutation of both S7 and S8 sites completely eliminated expression only within the shoulder but not elbow indicating that these two sites may cooperate to drive expression in the scapulo-humeral joint (Fig 3B). Finally, we also mutated two additional binding sites identified by MatInspector (PITX1 and ZEB1) and found no significant visible expression changes in shoulder, knee, and elbow compared to control constructs (S5 Fig, S1 Table). 


\section{Downstream regulatory sequences also contain multiple limb enhancers}

Since the Upstream BAC was not able to drive strong gene expression in distal joints, or in specific joints of the mid-limb, we also searched for additional non-coding sequences that were present in the Downstream BAC region (Fig 4A). We identified three non-coding regions evolutionarily conserved to chicken and located far downstream of Gdf5: R3 of $586 \mathrm{bp}$ at $+71 \mathrm{~kb}$ relative to the mouse $G d f 5$ coding region, $\mathrm{R} 4$ of $975 \mathrm{bp}$ at $+81 \mathrm{~kb}$, and $\mathrm{R} 5$ of $337 \mathrm{bp}$ at $+98 \mathrm{~kb}$ (Fig 4A, red boxes). We tested each in our transgenic assay at E14.5. R3 drove lacZ expression in the mesenchyme of the interdigital space and in digital transverse joint stripes as well as in the elbow and knee (Fig 4B, R3). R4 drove very strong expression in the joints of elbow, knee, digits, with the expression in the joints of shoulder and hip considerably weaker (Fig 4B, R4). R5 drove strong lacZ expression in the pre-chondrogenic mesenchyme of developing phalanges (Fig 4B, R5), and very weakly in the elbow and knee. One additional conserved region (GROW1 at $+45 \mathrm{~kb}$ ) drove expression in growth collars at ends of long bones rather than in developing joints. The possible role of GROW1 in controlling lengths of long bones is the subject of a separate manuscript (Capellini, Chen et al., in review).

The unique phalangeal pre-chondrogenic mesenchymal expression driven by R5 was not visible in embryos from the Downstream BAC construct, which covers both R5 and the other conserved regions tested above (Figs 1 and $4 \mathrm{~B}$ ). To explore this observation further, we concatenated R3, R4, and R5 and tested them as a single construct. Interestingly, we found they controlled expression in the same pattern as the Downstream BAC, i.e., within proximal limb joints of the shoulder, elbow, hip, and knee as well as distal joints of digits (Fig 4B, R3+R4+R5). As shown by histological sections, R3+R4+R5 collectively drove strong expression in the transverse stripes of the distal digit joints, along the lateral and medial joint capsules, and within the interdigital space (Fig 4B, arrows). However, the overall digit mesenchymal expression pattern of $\mathrm{R} 5$ was not reproduced by this construct, suggesting that R3 or R4 may act as a repressor element for the digit mesenchyme expression of R5. The possible trans-acting factors mediating this repressive effect are still unknown, but could be searched for in the future once the cis-acting elements are further narrowed. We also note that the overall expression driven by the composite $\mathrm{R} 3+\mathrm{R} 4+\mathrm{R} 5$ construct was both stronger and more reflective of the complete $G d f 5$ expression pattern than the pattern driven by the Upstream BAC (Fig $1 \mathrm{~B}$ and $1 \mathrm{C}$ ). Sections through the elbow joint indicate that the R3+R4+R5 construct drove gene expression in both the humeroradial (h-r) and humeroulnar (h-u) joints, consistent with the pattern of R4 (S4 Fig) and the Downstream BAC, but unlike the Upstream BAC (Fig 1B and 1C). Therefore, these three evolutionarily conserved non-coding regions located far from $G d f 5$ contribute to additional sites of expression in joints and long bones (S1 Table). Several of these expression sites are not seen with Upstream control elements, while others are not readily apparent by testing only large genomic sequences (i.e., BACs).

\section{Partial or complete rescue of Gdf5 mutant phenotypes by upstream and downstream BAC sequences}

To test the functional capacities of upstream and downstream regulatory domains, we took advantage of the fact that functional copies of the Gdf5 coding region are present within both of the Upstream and Downstream BAC clones. Transgenic mice carrying each BAC were bred onto a brachypodism ( $b p$ ) mutant background, followed by detailed examination of anatomical sites typically altered in $G d f 5$ mutant animals.

Hindlimbs of brachypodism homozygous mutants usually show fusions of tarsal bones or supernumerary bony elements in the ankle region, reduced length of metatarsals, absent 


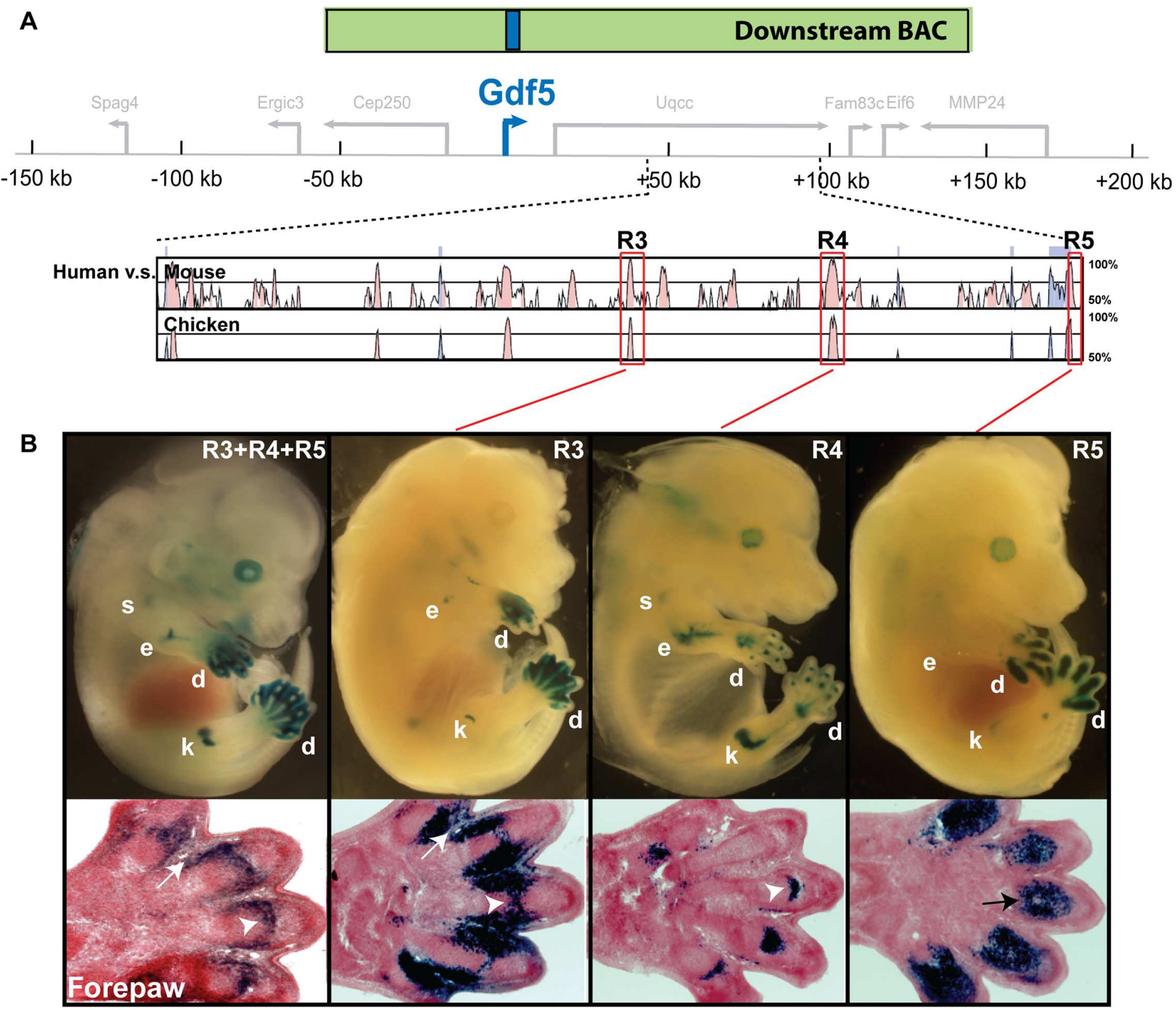

Fig 4. A cluster of three downstream regulatory enhancers interact to control gene expression in distinct distal limb tissues. (A) Three evolutionarily conserved elements (red boxes) within the Downstream BAC (green) region were identified via a comparison of mouse, chicken, and human sequences. Pink peaks represent conserved non-coding sequence showing at least $70 \%$ nucleotide identity over a $300 \mathrm{bp}$ window, while blue peaks reflect conservation in coding sequence. (B) Transgenic embryos collected at E14.5 from constructs containing either all three conserved noncoding elements as a concatenate (R3+R4+R5) (left) or individual regulatory elements (R3, R4, or R5 as indicated). Panels below each embryo are histological sections of the forepaw digital domains. When combined, all three elements (R3+R4+R5) drive weak expression in proximal limb joints such as the shoulder (s) and strong expression in the distal limb joints, such as the elbow (e), knee (k), and digits (d), along with the interdigital space (white arrow). R3 by itself drives expression in distal joints but also strongly in the interdigital space (white arrow) and interphalangeal joint (white arrowhead); R4 drives expression in distal limb joints including the interphalangeal joint (white arrowhead); and R5 drives expression weakly in distal limb joints, yet strongly in the phalangeal mesenchyme of the digits (black arrow).

doi:10.1371/journal.pgen.1006454.g004

middle phalanges on digits II-V, and much shorter digits (Fig 5) [7, 15]. The presence of the Upstream BAC restored normal formation in tarsal joints and ankles, and improved metatarsal lengths compared $b p / b p$ mutants (Fig 5A, left). However middle phalanges were missing in Upstream BAC-positive mice, and digits were still substantially shorter than wild type. Thus, normal bone and joint patterning in digits is not rescued by Upstream BAC sequences. 


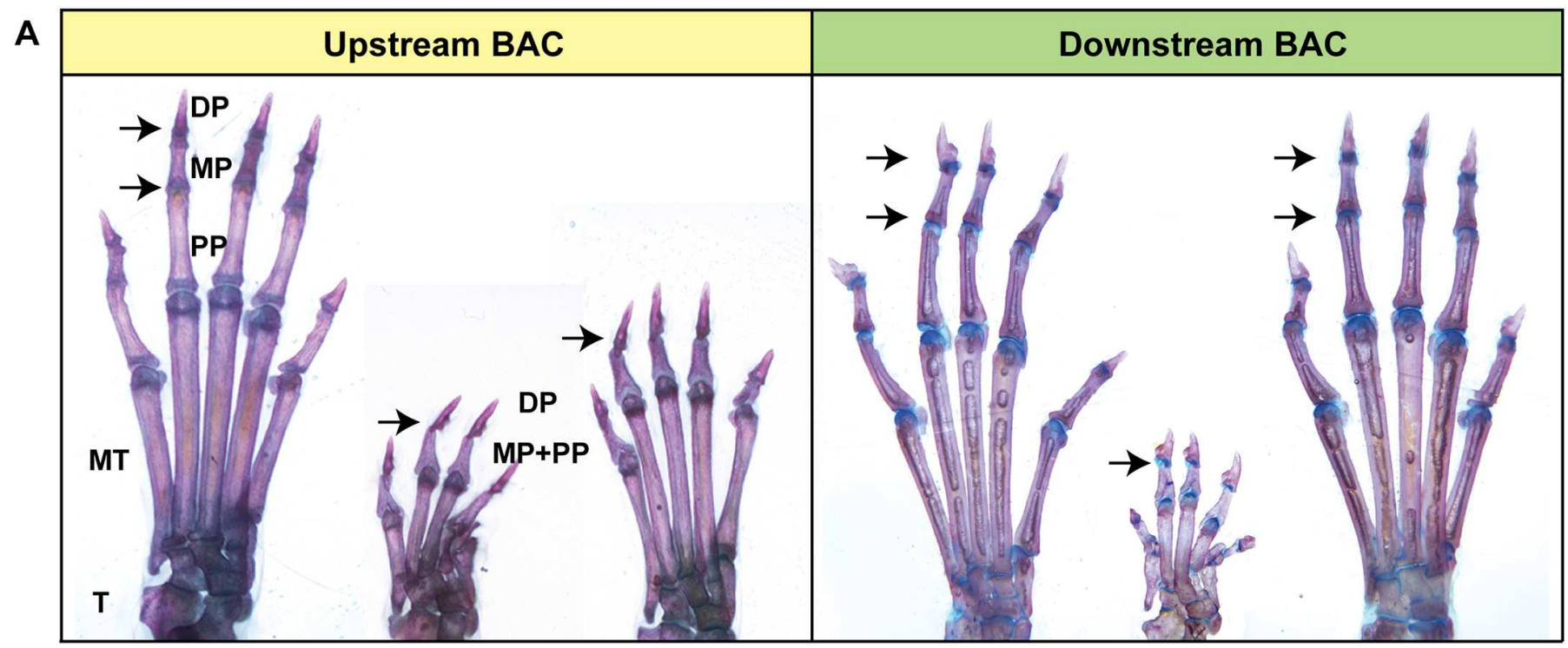

$b p /+$, BAC- $\quad b p / b p$, BAC- $\quad b p / b p$, BAC+

$b p /+$, BAC- $\quad b p / b p$, BAC- $\quad b p / b p, B A C+$

B

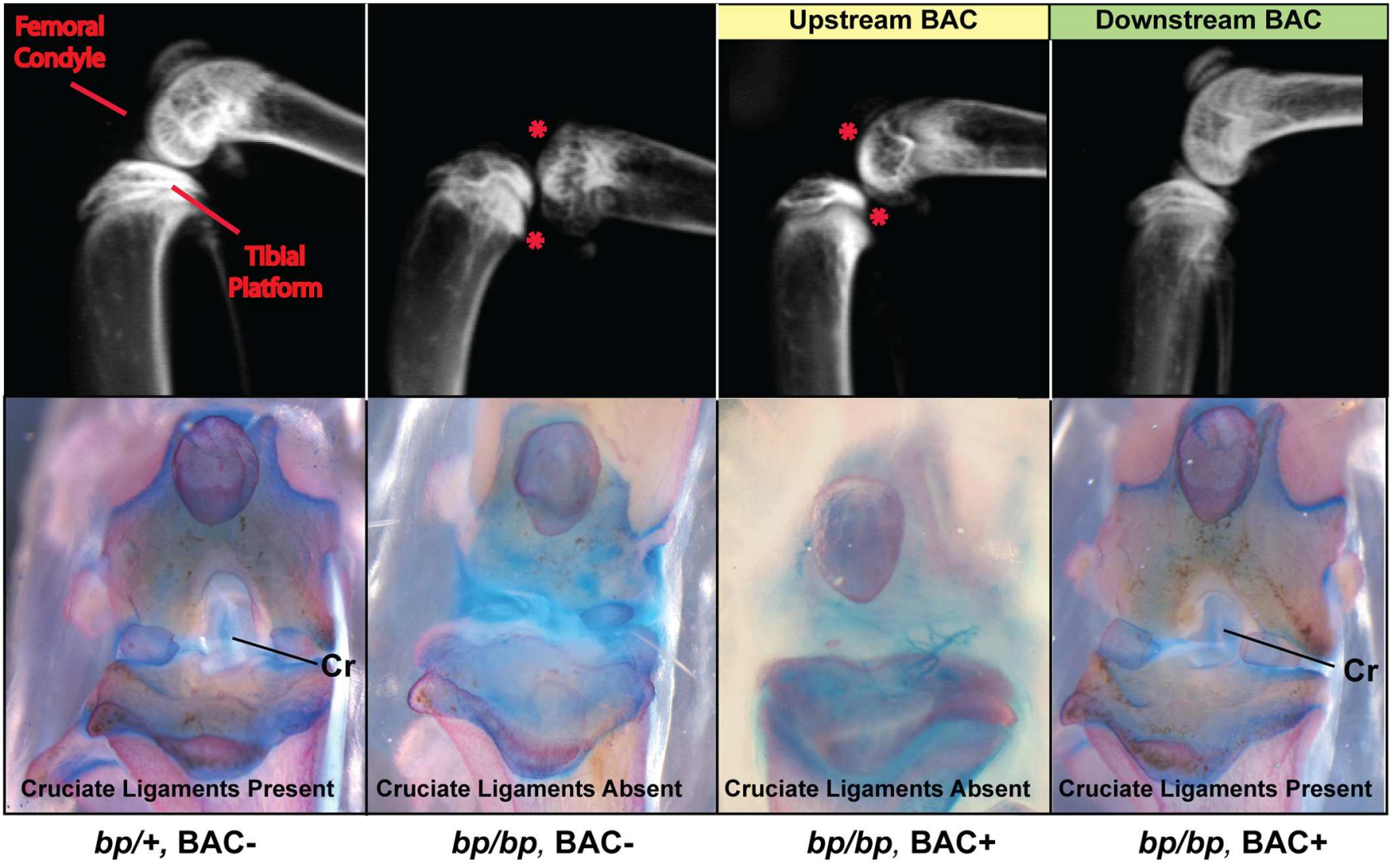

Fig 5. Downstream Gdf5 regulatory regions are required for full rescue of brachypodism joint defects. (A) Skeletal preparations of hindpaws of transgenic rescue experiments with either Upstream (yellow) and Downstream (green) BACs. Control mice (bp/+, BAC-; left) are shown in comparison to brachypodism homozygous mutants that are either BAC negative ( $b p / b p, B A C$-; center) or are also carrying either Upstream or Downstream BAC positive ( $b p / b p, B A C+$; right). In each panel digit I is to the right. DP (distal phalange), MP (middle phalange), PP (proximal phalange), MT (metatarsal), and T (tarsal). Arrows indicate location of the interphalangeal joints. (B) (Top) Radiographs of adult mouse knee joints. Asterisks ( $\left.{ }^{*}\right)$ indicate the femoral and tibial dysmorphologies typical of $b p / b p$ mutants. (Bottom) Alcian Blue (cartilage) and Alizarin red (bone) preparations of adult knees from rescue experiments. Cruciate ligaments $(\mathrm{Cr})$ are usually missing from $b p / b p$ mice. Note that the presence of the Downstream $\mathrm{BAC}(\mathrm{bp} / \mathrm{bp}, \mathrm{Downstream}$ $B A C+$ animals) fully restores interphalangeal joints, adult knee joint articular morphology, and cruciate ligaments; while the Upstream BAC does not.

doi:10.1371/journal.pgen.1006454.g005 
In contrast, the Downstream BAC sequences fully restored multiple phenotypes in $b p / b p$ mutants. BAC-positive mice showed normal tarsals and ankles, metatarsal and phalangeal lengths, and possessed the middle phalanges in digits II-V (Fig 5A, right).

In addition to possessing dysmorphic and shorter paws than wild-type mice, $b p / b p$ mutants develop knee OA when experimentally challenged [18] and consistently display dislocated joints with poorly developed femoral and tibial articular surfaces and absent cruciate ligaments (Fig 5B) [17]. The Upstream BAC partially restored distal femoral condylar morphology but was unable to restore the proper registration of the femur and tibia, or rescue the absence of the anterior and posterior cruciate ligaments (Fig 5B). In contrast, the Downstream BAC allele rescued multiple knee phenotypes, leading to proper registration between femur and tibia, normal morphology of joint surfaces, and restored cruciate ligaments (Fig 5B). Thus, although Upstream BAC sequences can partially rescue some Gdf5 phenotypes, including normal joint formation in ankle regions, additional sequences in the Downstream BAC are required to rescue joint formation in distal digit regions, and to restore knee surfaces and ligaments.

\section{Discussion}

We have identified a series of cis-acting regulatory elements distributed upstream and downstream of the Gdf5 gene spanning an approximate $100 \mathrm{~kb}$ interval in mice. Previous studies showed that an upstream BAC clone could recapitulate multiple aspects of $G d f 5$ expression, including expression in multiple joint interzones [13]. Although the upstream BAC clone can be used to drive Cre expression and modify the activity of other genes in joints [13], additional downstream regulatory sequences are clearly required to achieve full expression in limbs, to fully rescue normal bone and joint formation in digits, and to restore knee structures in brachypodism mutant mice.

Separable enhancers within both the upstream and downstream regions show a striking degree of anatomical specificity, including: 1) different anatomical divisions within the body (e.g., appendicular skeleton, axial skeleton, cranium); 2) different skeletal tissue types (e.g., articular cartilage, interdigital mesenchyme, pre-cartilaginous mesenchyme); 3) different limb types (e.g., forelimb, hindlimb); 4) different domains within the limb (e.g., proximal limb, distal limb); and 5) different individual joints within limbs (e.g., elbow versus knee; humeroradial versus humeroulnar joints) (Fig 6, upper panel; S1 Table). If and how these sequences regulate additional genes in their vicinity is currently unknown. We note, however, that the centrosomal protein 250KD (Cep250) gene and the ubiquinol-cytochrome c reductase complex assembly factor 1 (Uqcc1) genes, which map upstream and downstream of Gdf5, do not show the striking joint expression patterns that are shared by Gdf5 and the various joint enhancers reported here (S6 Fig).

In terms of overall regulatory architecture, our results are reminiscent of previous studies of repeating stripes of gene expression during early Drosophila development. In Drosophila, multiple pair-rule genes are expressed in seven characteristic stripes in early embryos [45]. For "secondary" pair rule genes, transcriptional control of the seven-stripe pattern depends upon on earlier "primary" pair rule genes. In contrast, transcriptional control of "primary" pair rule genes like hairy and even-skipped (eve) is considerably more complex [46, 47]. For example, multiple distinct enhancers in the eve locus control expression only in particular stripes or subsets of stripes, with specific enhancers responding to different types and concentrations of upstream transcription factors that are found at particular anatomical positions along the anterior-posterior axis [48]. The simple appearing multiple-stripe pattern of the eve locus is thus built from a composite of underlying mechanisms, and several different enhancers within the gene are required to reproduce the overall pattern [49]. 


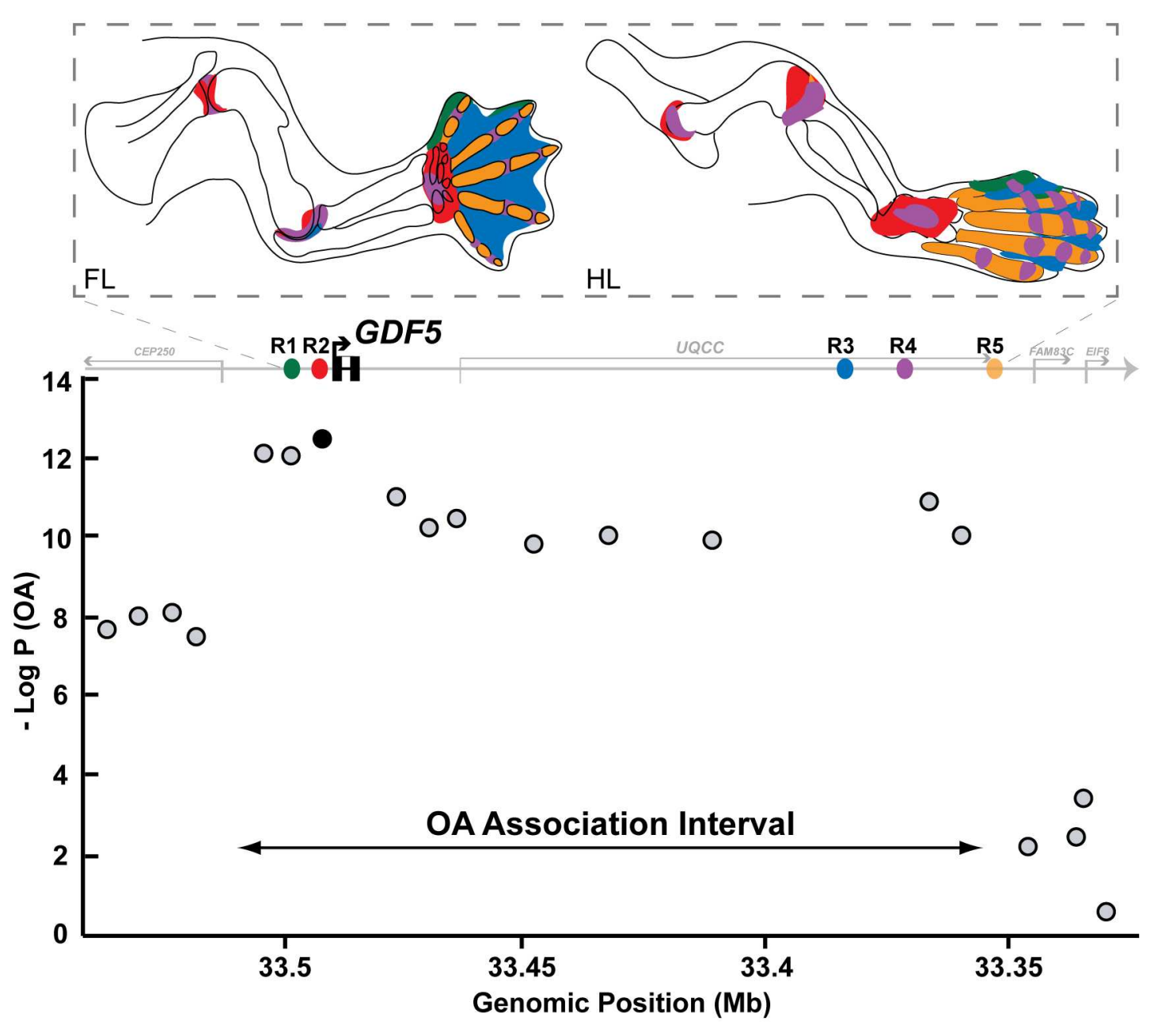

Fig 6. Modular regulatory architecture of GDF5 spans the region linked to Osteoarthritis (OA) susceptibility in humans. (Top) Summary of the different stripes and anatomical domains controlled by separate regulatory enhancers (colored) in the Gdf5 gene in E14.5 developing mouse forelimbs (FL) and hindlimbs (HL). (Bottom) Association of various human SNPs (grey circles) with adult knee OA in cases vs. controls (based on [27]). Y-axis is the -log P-value of the trait association for SNPs across the interval. X-axes show genomic megabase locations (bottom axis) of human sequences orthologous to R1 (green), R2 (red), R3 (blue), R4 (purple), and R5 (orange) elements (top axis). The highest scoring variant tested in the human study, rs143383 (dark circle), is located in GDF55'UTR, immediately downstream of the R2 region. Note that significant association extends over a broad region, and many linked human variants have not yet been tested, including common human variants in R2, R3, R4, and R5 (see S4 Table).

doi:10.1371/journal.pgen.1006454.g006

Our results suggest that the vertebrate $G d f 5$ locus is controlled in a similar piecemeal fashion. Gdf5 is expressed in a striking pattern of stripes that are seen almost everywhere that synovial joints form in the head, vertebral column, and limbs (Fig 6, upper panel). Although the regularity of the stripe expression in interzones might have been controlled by a common regulatory mechanism, different joint stripes are clearly controlled by separate regulatory enhancers which are distributed over large genomic regions both upstream and downstream of $G d f 5$ coding sequences. At least one additional enhancer in the Gdf5 downstream region is expressed in collars near the ends of bones, and may be involved in regulating long bone growth rather than joint formation or maintenance (Capellini, Chen et al., in review). Multiple enhancers are required not only to recreate the gene's overall expression pattern in embryos, but also to rescue limb phenotypes seen in Gdf5-deficient mice, 
confirming the importance of the distributed cis-acting control sequences for overall Gdf5 function.

Unlike the case in Drosophila, the upstream factors that regulate expression from individual Gdf5 enhancers are still largely unknown. Multiple binding sites can be predicted in the sequences of the R1, R2, R3, R4, and R5 elements identified in this study, based on large databases of binding interactions between transcription factors and target sequences (UNIPROBE, [50]), as well as large-scale chromatin-immunoprecipitation (ChIP-seq) experiments from cell lines and embryos. However, interzones are transient structures that are not recapitulated in immortal cell lines. They also make up only a small fraction of cells in developing embryonic structures, and so are not well represented in bulk studies of transcription factor binding in developing tissues. Given the relatively large size of the $G d f 5$ enhancers as defined by functional studies, the small size of most consensus binding sites, and the relative paucity of interzone cells in most large-scale chromatin interaction studies, many relevant factors controlling specific stripes of Gdf5 expression likely remain to be discovered.

Despite these difficulties, some candidate upstream regulators can be postulated, based on genes that are required for normal joint development, or that are differentially expressed in particular limbs or different joints within the limb, including WNT signals, and members of the SOX, HOX, TBX, DLX, PITX, and ZEB1 transcription factor families [51-65]. Predicted PITX1 and ZEB1 binding sites are present in the R2 enhancer element, and the known hindlimb-specific expression of PITX1 would appear to be a promising mechanism for controlling hindlimb-specific stripes of Gdf5 expression. However, mutating either PITX1 or ZEB1 binding sites did not significantly alter R2d+e enhancer joint patterns in the limbs (S5 Fig, S1 Table), suggesting that additional binding sites or other upstream factors are likely involved.

Members of the BARX family of transcription factors are also expressed in interzone stripes, a pattern that closely resembles that of Gdf5. Barx2 null mice show altered joint formation but do not lose Gdf5 expression [41]. However, Barx1 is also expressed in limbs and joints $[66,67]$, and functional redundancy among BARX family members may make it difficult to detect the full range of Barx phenotypes in developing joints. Given the overlapping expression patterns of Barx2 and Gdf5, we tested the effects of mutating specific BARX2 binding sites in the R2 enhancer, though the predicted sites may also bind additional factors. Individual mutation of some sites (i.e., S4, S5, S7) had no visible effect on joint-specific enhancer activity as assayed by lac $Z$ expression. However, mutation of site $\mathrm{S} 6$ significantly reduced expression within the knee, whereas mutations of both S7 and S8 reduced shoulder expression. It is possible that our site-specific mutations disrupt binding sites for other upstream factors in addition to BARX2, including other homeodomain factors that bind similar sequences. Nonetheless, our results clearly identify specific small sequence motifs within the R2 enhancer that are required for expression in individual joints in forelimbs or hindlimbs. Future studies of these and other enhancer sequences should help elucidate mechanisms controlling expression in specific joints in the vertebrate skeleton.

\section{Evolution of Gdf5 control sequences}

Gdf5 is expressed in synovial joints from a wide range of species, including fish, birds, and mammals $[9,11,21,29,68,69]$. Comparative sequence analysis shows that the 5 ' R2 regulatory element controlling strong proximal limb joint expression is conserved from mammals to at least Danio rerio (Zebrafish), while the $3^{\prime}$ distal limb element cluster (R3+R4+R5) is conserved only between mammals and Xenopus tropicalis (Western clawed frog). The 5 ' joint control element may be older (or more constrained) than the joint elements in the 3' distal cluster, perhaps because $\mathrm{R} 2$ is required to build the more ancient proximal fin skeleton of aquatic 
vertebrates. Conversely, the distal 3 ' regulatory elements, which control expression not just in joints but in lateral/medial chondrogenic tissues, interdigital space, and developing phalangeal element mesenchyme, may have evolved during the later emergence of distal limb structures in terrestrial vertebrates. Such additive modular control has been posited for other organs. For example, $N k \times 2-5$ has multiple distinct cis-acting elements that direct transcription specifically in different sub-regions of the developing heart [70-73] and this complexity may have played an important role in the evolution of a multi-chambered mammalian heart [74].

Modular regulatory architectures may be particularly important for genes that play roles in the development of highly patterned structures that have distinct sizes and shapes at particular anatomic positions in different species, such as skeletons and muscles. Other BMPs involved in cartilage and bone formation, such as Bmp5 and Gdf6 [75-77] as well as members of the $M y o D$ and $M r f$ families that control muscle determination [78, 79] are all controlled by complex sets of highly specific modular enhancers. Gain, loss, or modification of anatomically specific enhancers within such genes may be a common way of altering particular structures in the body during evolution, while preserving other essential aspects of gene function [80-83].

\section{Implications for human health and disease}

Multiple human congenital skeletal defects have previously been traced to coding region changes in the GDF5 gene, including: acromesomelic chondrodysplasia Hunter-Thompson syndrome [20, 21], Grebe syndrome [22], Brachydactyly Type A2 and C and synostoses [84, 85].

More recently, common forms of OA have also been linked to genetic variation around the GDF5 locus, in the absence of protein coding changes. Miyamoto et al. (2007) was the first to report that derived SNP variants "T" at rs143383 and "T" at rs143384 in the 5'UTR region of GDF5 are significantly associated with common hip and knee OA in Japanese and Chinese populations (1.5-2.1 OR) [27]. This finding was confirmed in Europeans [29], and many subsequent studies have reported significant associations in Eurasians between the same SNPs and OA in different joints such as the hand, hip, and knee (selected examples include [86-92]).

Reconstruction experiments have shown that the derived " $\mathrm{T}$ " risk alleles at rs 143383 and rs143384 reduce quantitative levels of gene expression when transfected with reporter genes into tissue culture cells in vitro $[27,33]$. Additionally, a rare cis-acting promoter variant has been shown to modulate the activities of both "T" variants in similar transfection assays [33]. Although both common "T" variants are actually located in the transcribed 5'UTR region of GDF5, they are closely linked to the R2 upstream enhancer region identified here. To test for possible qualitative effects of these SNPs on joint expression patterns in vivo, we cloned both the derived "T,T" risk variants and the ancestral "C,C" protective variants of rs143383 and rs143384 into separate 1468 base pair constructs carrying the R2 enhancer upstream of the Hsp68 basal promoter and a lac $Z$ expression cassette. We did not observe any visible difference in expression within the different joints of the limb when comparing transgenic mouse embryos made with the different constructs (S7 Fig, S1 Table). Subtle expression differences would be difficult to detect with this assay, and it possible that more substantial differences would be detected at different time points or in the presence of other disease associated mutations.

Interestingly, the genomic region significantly associated with OA risk in humans actually extends substantially beyond the rs143383 and rs143384 SNPs in the 5'UTR of the gene (Fig 6, bottom panel) [27]. A $130 \mathrm{~kb}$ risk haplotype is present at high frequency in Eurasian populations. Markers throughout this region are in high linkage disequilibrium with the 5 'UTR 
SNPs, and show similarly high association with OA phenotypes in studies that have tested additional markers [27, 86-92]. We note that the human orthologs of the R2, R3, R4, and R5 enhancer elements are all located within this broader haplotype and $\mathrm{OA}$ association interval. Based on chromatin accessibility and modification in human chondrocytes and developing embryonic limbs, the orthologous human non-coding regions, along with additional regulatory sequences, also appear to be active in relevant tissues and time points during joint development (S8 Fig). Importantly, our functional experiments also reveal that the 3' downstream regions of the mouse $G d f 5$ locus, rather than the 5 ' upstream regions, are capable of driving postnatal expression in knee articular structures (S3 Fig). In addition, it is the Downstream but not Upstream BAC that is capable of fully restoring articular structures and restoring knee ligaments in Gdf5 mutant mice (Fig 5). Many common variants are located within the syntenic downstream region of the human GDF5 locus, including common variants found in the human orthologs of the R3, R4, and, R5 enhancers (S4 Table), and likely in sequences that remain unidentified by our approach, including sequences that are located even further upstream or downstream of the gene. While humans may have additional GDF5 enhancers not yet identified in the mouse studies, the human orthologs of mouse Gdf5 enhancers are a promising place to look for possible functional non-coding mutations that may alter GDF5 expression, joint structures, and disease risk in human populations, including changes that are specific to particular subsets of joints in the skeleton.

\section{Materials and Methods Ethics statement}

All experiments performed on adult, fetal, and embryonic mice including euthanasia have been approved by the Stanford University Institutional Animal Care and Use Committee (IACUC) and were performed in accordance with Stanford Administrative Panel on Laboratory Animal Care Guidelines (approved protocol 10665), in facilities certified by the American Association of Laboratory Animal Science. No human subjects were utilized in this study. No field permits were required, granted, or utilized for this study.

\section{BAC modifications}

The Upstream Gdf5-BAC was isolated from a Research Genetics 129Sv BAC library by PCR screening with specific primers from the Gdf5 3'UTR (5'-CGACTCTGCCAACAACGTGG-3' and 5'-CACCTTTCCTGAGCCCCAGG-3'). The size and orientation of the BAC inserts were determined using restriction mapping, pulse-field gel electrophoresis, and Southern analysis. The Downstream BAC RP23-316K12 was identified by searching published mouse BAC-end sequences for those that extend further downstream of the first Upstream BAC.

The two BACs were modified as described previously [93]. A Gdf5 targeting cassette was made by inserting 5 ' and 3' homology arms into a recombination vector pIRES- $\beta$ Geo-Ftet, which contains an IRES- $\beta$ Geo cassette and a tetracycline resistance cassette flanked by FRT sites. The PCR primers for amplifying the homology arms were (5' Arm: forward with NheI linker 5'-GGATTGCTAGCTATTCATCGACTCTGCCAACAACGTGG-3' and reverse with XhoI linker 5'-GGATTCTCGAGTAAGCAGCTTCACAGGCTCTCTGTTAC-3'. 3' Arm: forward with SpeI linker 5'-GCATGACTAGTGCTGCTGCCCGAAGTTTCCTGG-3' and reverse with NotI linker 5'-GGATTGCGGCCGCTAAAGAACACCTTTCCTGAGCCCCA GG-3'). BAC modifications were carried out by homologous recombination with the targeting cassette in EL250 bacteria strain containing the original Gdf5-BACs. Successfully targeted BACs were verified by sequencing, and contained an IRES- $\beta$ Geo cassette inserted within the 
3'UTR of the gene, $747 \mathrm{bp}$ downstream of the Gdf5 stop codon. The tetracycline resistance gene was subsequently removed by induction of Flpe recombinase.

\section{Hsp68lacZ plasmid constructions}

Evolutionarily conserved non-coding regions (ECR) to be tested for enhancer activity were PCR amplified with primers containing NotI restriction sites, and cloned into the p5'NotIhsp68 lacZ expression vector containing a minimal heat shock promoter and the lac $Z$ cassette [94]. For constructs where concatenated copies were required, primers containing Sfil sites were used and the PCR products were ligated briefly to form tandem copies before cloning into a modified p5'NotI-hsp68 lacZ vector with a SfiI restriction site inserted between two NotI sites upstream of the hsp68 lacZ cassette [75]. All primers used to amplify ECRs described in this study are listed in S5 Table.

\section{Transgenic mice}

Transgenic mice were generated by pronuclear injections carried out either by Michael Schoor, the Stanford Transgenic Facility, or Taconic/Xenogen Biosciences. All constructs were linearized and then were purified for microinjection into FVB or C57BL6/CBA F1 fertilized oocytes as previously described $[94,95]$. To facilitate analysis of many different constructs, most transgenic embryos were collected at E14.5 for X-gal staining, without further breeding. For each construct, multiple transgenic embryos derived from independent integration events were analyzed, and only patterns seen consistently in 3 or more independent embryos are reported. Please refer to $\underline{\mathrm{S} 1 \mathrm{Table}}$ for a summary of the number of transient transgenic embryos generated for each construct, and the expression patterns seen. For the Upstream and Downstream BAC constructs, stable transgenic lines were also generated by allowing injected embryos to come to term, and outcrossing to transmit the transgene. Multiple stable lines were used to confirm E14.5 day expression patterns (S1 Table), and to analyze expression and phenotypic rescue in adult mice.

\section{X-gal staining and sections}

Whole mount staining for $\beta$-galactosidase activity was performed as described with minor modifications [94]. Embryos were fixed for 45 minutes in fresh $4 \%$ PFA in PBS at $4^{\circ} \mathrm{C}$, cut in half and then fixed for additional 15 minutes. Fixed embryos were washed 3 times in wash buffer and stained for 16-24 hours in the dark with $1 \mathrm{mg} / \mathrm{ml} \mathrm{X-gal} \mathrm{in} \mathrm{staining} \mathrm{buffer} \mathrm{at} \mathrm{room}$ temperature. After staining, embryos were briefly washed in wash buffer and post-fixed in $4 \%$ PFA for 5 hours. Times were adjusted accordingly for E17.5 and post-natal specimens. For sectioning, X-gal stained embryos were placed first in sucrose and then embedded in gelatin/ sucrose solution and cryo-sectioned at $25 \mu \mathrm{m}$. Sections were counterstained with Nuclear Fast Red (Vector labs, \#H-3403).

\section{In situ hybridization}

Antisense and sense digoxigenin-labeled probes for in situ hybridization were generated for Gdf5, Cep250, and Uqcc1. Gdf5 probes were generated as described [7]. Cep250 probes were generated by amplifying a 1593bp fragment corresponding to the 3'UTR from mouse genomic DNA using primers 5'- TTGCCAGAAGAAAGAAGAGCTGAGG-3' and 5'-TTTATTGTC GAAGGGAAGATGAGGG -3'. The fragment was next cloned into pBluescript SK vector, and sense and antisense probes were produced by digesting with EcoRI and transcribing with T3 and T7 RNA polymerase, respectively. Uqcc1 probes were generated by amplifying a 1074bp 
fragment corresponding to the 3'UTR from mouse genomic DNA using primers 5'-TTCACT CAGAAACCCCTGTGCTTGG -3' and 5'- TGCCCAGATGTAATGAGTTACAAGG-3'. The fragment was then cloned into pBluescript SK vector, and sense and antisense probes were produced by digesting with EcoRI and transcribing with T3 and T7 RNA polymerase, respectively. Next, to compare Gdf5 lac Z expression to endogenous gene expression for each of these genes, E15 Downstream BAC embryos were harvested, next snap-frozen and then embedded in Tissue-Tek OCT compound. Embedded embryos were then serially sectioned on a cryostat. Adjacent sections were either stained for lac $Z$ expression using the X-gal staining methods described above, or used in standard in situ hybridization protocols as described [96].

\section{Phenotypic rescue experiments}

Three independent mouse lines per Upstream BAC or Downstream BAC were each crossed to animals homozygous for $b p^{J}$ or $b p^{3 J}$ brachypodism alleles, respectively. The $b p^{J}$ mutation occurred spontaneously in $\mathrm{A} / \mathrm{J}$ mice, and $b p^{3 J}$ in $\mathrm{BALB} / \mathrm{cJ}$. Both mutations result in frameshifts and premature translational termination in the Gdf5 open reading frame [7]. Gdf5-BAC/+; $b p /$ + animals were crossed to non-transgenic $b p / b p$ mice, and progeny were genotyped for the lac $Z$ transgene and brachypodism mutations in separate PCR reactions. The primers used for lacZ genotyping were: 5'-TTTCCATGTTGCCACTCGC-3' and 5'-AACGGCTTGCCGTTCA GCA-3'. The brachypodism mutations were genotyped with primers that amplify the endogenous $G d f 5$ locus but not the Gdf5-BAC transgene (due to the presence of the IRES- $\beta$ Geo insertion; forward 5'-ACCTGGAACTCATCTGCACTGTG-3' and reverse 5'-TGGGAAACAGT TATACCTGAGG-3').

Eight-week old female Gdf5-BAC/+; bp/bp mice, +/+; bp/bp mice, and +/+; bp/+ mice were stained with Alcian blue (cartilage) and Alizarin red (bone) and cleared as described [97]. Specifically, the skin and visceral organs were removed and the animals were fixed in $95 \%$ ethanol. The specimens were then stained in a mixture of Alcian blue, acetic acid, and ethanol. After several washes in $95 \%$ ethanol, the samples were cleared in $\mathrm{KOH}$ and then stained by Alizarin red in $\mathrm{KOH}$ solution. The skeletons were stored and photographed in glycerol. Knees and hindpaws were examined and imaged under a light microscope for joint morphology and the presence/absence of the cruciate ligaments. For radiographic analysis, intact hindlimbs were positioned laterally on Kodak radiographic film and placed in a Faxitron X-ray system at $18 \mathrm{kV}$ and $0.3 \mathrm{~mA}$, for 22 seconds. For each group of animals, homozygous $b p$ animals without the $\mathrm{BAC}$, homozygous $b p$ animals with the $\mathrm{BAC}$ and heterozygous $b p$ animals without the $\mathrm{BAC}$, $\mathrm{n}=8$.

\section{Comparative sequence analysis}

Sequence data for different species was obtained by searching publicly available nucleotide databases at NCBI (http://www.ncbi.nlm.nih.gov/BLAST/). Evolutionarily conserved noncoding sequences were identified using global sequence alignment programs including:

PipMaker (http://bio.cse.psu.edu/pipmaker),

VISTA (formerly at http://genome.lbl.gov/vista/index.shtml, [98]), and LAGAN (http://lagan.stanford.edu/lagan_web/index.shtml, [99]).

\section{Predicted transcription factor binding sites}

To find upstream transcription factors predicted to bind to R2 regulatory sequences, we used Vista with MatInspector, and UNIPROBE. rVista uses the TRANSFAC database to look for potential binding sites that are highly conserved across sequences [38, 39]. MatInspector of the Genomatix suite (http://www.genomatix.de) uses a large library of matrix descriptions for 
transcription factor binding sites to find corresponding matches in a target sequence [37, 40]. The UNIPROBE database (http://the_brain.bwh.harvard.edu/uniprobe/about.php) is built on replicate experimental measurements of binding affinities between large numbers of expressed transcription factors and all possible 8-mer target oligonucleotides [50, 100]. At the recommended enrichment threshold of 0.4, UNIPROBE identified over 3,000 sites (i.e., specific 8 -mer sequences bound by a transcription factor) in mouse and human R2 sequences. The relevant factors, 8-mers (and reverse complements), positions in the R2 sequence, and enrichment scores depicting relative binding affinity for each factor are summarized in S2 Table, sheets $1-2$.

Lists of potential upstream regulators were intersected with expression and phenotypic data in order to identify those transcription factors also known to be expressed or required in limbs and joints, based on data in VisiGene (http://genome.ucsc.edu/cgi-bin/hgVisigene), Eurexpress (http://www.eurexpress.org/ee/), Genepaint (http://www.genepaint.org/Frameset.html), and the Mouse Genome Informatics expression and phenotypic databases (http://www.informatics.jax. org). Barx2 displayed overlap with known Gdf5 expression patterns, specifically at gestational days when R2 enhancer was active [41-44]. Pitx1 is expressed in hindlimbs and is required for normal development of knees [55]. Zeb1 is also expressed in joints, and null mice exhibit multiple skeletal defects, including the fusion of the humerus to either radius or ulna [53].

Engineered R2 sequences carrying site-specific mutations in predicted BARX2 binding sites were synthesized by GenScript. S3 Table shows the calculated changes in UNIPROBE enrichment scores for BARX2 binding to wild type and mutated sites. $2 \mathrm{X}$ copies of wild type and mutant R2 sequences were cloned into the Hsp68 lacZ reporter with appropriate primers and restriction enzyme sites (S5 Table), and used to generate multiple independent E14.5 transgenic mouse embryos for lacZ expression analysis as above (S1 Table).

A three-base pair mutation was engineered in the single ZEB1 binding site and the single PITX binding site in the R2d+e region, based on presence and loss of binding sites in MatInspector. Altered R2d+e constructs were synthesized by PCR-sequence overlap extension (PCR-SOEing) [101] using the primers listed in S5 Table. Wild type and mutant constructs were and used to generate E14.5 transgenic mouse embryos as above (S1 Table). Neither binding site mutation produced a noticeable alteration in the limb joint expression patterns driven by the unaltered $\mathrm{R} 2 \mathrm{~d}+\mathrm{e}$ construct.

\section{Supporting Information}

S1 Table. Summary of expression patterns driven by BACs or smaller constructs in independent E14.5 transgenic embryos. (XLSX)

S2 Table. UNIPROBE analysis of transcription factor binding sites in human and mouse $\mathrm{R} 2$ sequences.

(XLS)

S3 Table. UNIPROBE analysis of engineered mutations in mouse $\mathrm{R} 2$ sequence. (XLS)

S4 Table. Summary of common human polymorphisms that map in non-coding orthologs of mouse Gdf5 enhancers.

(XLSX)

S5 Table. Primers used to make hsp68-lacZ transgene constructs. (PPT) 
S1 Fig. Upstream BAC transgenic embryos recapitulate many sites of endogenous Gdf5 expression in axial joints and connective tissues. In all panels the ventral side is left and anterior is at top. a, Side view of E14.5 Upstream BAC transgenic embryo showing lacZ expression in numerous anatomical locations (a, ankle; e, elbow joint; $h$, hip joint; $k$, knee joint; me, middle ear; r, ribs; s, shoulder joint; $w$, wrist) $\mathbf{b}$, Medial view of bisected embryo as in a, showing lac $Z$ expression internally (cp, choroid plexus; 1 , larynx; $n$, neck joint; st, sternum; t, tail ligament; vc, vertebral column). c, lacZ expression in tooth buds. d, lacZ expression in sternal joints. e, Endogenous $G d f 5$ expression in tooth bud (white arrowhead) and sternal joints (black arrowheads). $\mathbf{f}$, lac Z expression in joint between basioccipital bone (b) and atlas (at). In $\mathrm{f}$, the joint between the bodies of the atlas and axis is out of the plane of section ( $\mathrm{ax}^{*}=$ transverse process of the axis). g, Endogenous Gdf5 expression in basioccipital-atlas joint and atlasaxis joint ( $\mathrm{ax}=$ axis). $\mathbf{h}$, lac $Z$ expression in rib-vertebral joints (black arrowheads) and intervertebral joints (white arrowheads). i, Endogenous $G d f 5$ expression similar to lacZ expression shown in h. c, d, f, and h are sagittal cryosections of E14.5 Upstream BAC transgenic embryos stained by X-gal and counterstained with neutral red. e, g, and i show in situ hybridization with antisense $G d f 5$ probe to sagittal cryosections of nontransgenic E14.5 embryos.

S2 Fig. Expression of Upstream BAC transgene in adult mice. a, Cleared foot skeleton from a transgenic Upstream BAC positive adult 2 month old mouse, stained with Alizarin red and with X-gal, showing persistence of lacZ expression in joints. $\mathbf{b}$, Magnified view of a metacarpal-phalangeal joint showing lacZ expression on the articular surface. $\mathbf{c}$, Section through joint in $\mathrm{b}$ showing lac $Z$ expression in the superficial chondrocytes of the articular cartilage. $\mathbf{d}$,

Magnified view of vertebral articulation of Upstream BAC transgenic in a 2 month old adult mouse, stained as in a. Note lacZ expression at the sites of articulation. e, lacZ expression at the insertion site (arrowhead) of the Achilles tendon on the calcaneus.

S3 Fig. Comparison of Upstream and Downstream BAC expression patterns in pre- and post-natal knee structures. Xgal stained knee joints of mice harboring either the Upstream BAC (A-D)) or Downstream BAC (E-H) at late gestational stages (E17.5 embryos) (A, B, E, F) or post-natal 3 month old adults $(\mathbf{C}, \mathbf{D}, \mathbf{G}, \mathbf{H})$. At E17.5, regulatory elements within the Upstream BAC (A, B) drove moderate expression in the cruciate ligaments $(\mathrm{Cr})$ as well as the articular surfaces of the femur and tibia (denoted with an asterisk, ${ }^{*}$ ), whereas sequences within the Downstream BAC (E, F) drove strong expression in these domains, along with additional expanded locations such as the articular capsule (AC) and the medial and lateral meniscus (Me). At post-natal 3 months, end-on views are shown of the exposed articular surfaces of the femur $(\mathbf{C}, \mathbf{G})$ and of the tibia $(\mathbf{D}, \mathbf{H})$. Note that regulatory sequences within the Downstream BAC continue to drive strong expression in the articular surfaces of the femur (i.e., patella groove, PG; femoral condyle, FC) and the tibia (i.e., tibial platform, TP), with expression persisting in the meniscus (Me, lateral meniscus removed to expose $\mathrm{TP}$ ) and collateral ligaments (CL) (panels $\mathbf{G}, \mathbf{H}$ ). These latter post-natal patterns were not observed in mice carrying the Upstream BAC reporter (panels C, D).

S4 Fig. Expression patterns driven by R2, R3+R4+R5, and R4 constructs. (A) Four evolutionarily conserved elements (red boxes) within the Upstream (yellow) and Downstream (green) BACs were identified via a comparison of mouse, chicken, and human sequence conservation. Pink peaks represent conserved non-coding sequence showing at least $70 \%$ nucleotide identity over a $300 \mathrm{bp}$ window, while blue peaks reflect conservation in coding sequence. 
(B) First row: representative transgenic embryos showing lacZ expression patterns driven by $\mathrm{R} 2, \mathrm{R} 3+\mathrm{R} 4+\mathrm{R} 5$, and $\mathrm{R} 4$ constructs. Second row: forepaw expression in these embryos. Third and fourth rows: serial sections of elbow joints. Note that R2, R3+R4+R5, and R4 drove lacZ expression in proximal synovial joints, such as shoulder (s), elbow (e), and knee (k), as well as distal joints of wrist (w), ankle (a), and digit joints (d), although for the R2 element, digital expression was inconsistent (i.e., less than half of lacZ positive embryos exhibited this expression pattern). $\mathrm{R} 3+\mathrm{R} 4+\mathrm{R} 5$ drove strong digit ( $\mathrm{d}$ or white arrowhead) and interdigital space (white arrow) lacZ expression, while R4 only drove joint expression (white arrowhead). In contrast to R2, which only drove lacZ expression in humeroradial (h-r) joint, R3+R4+R5 drove expression in both humeroradial (h-r) and the humeroulnar (h-u) joints. R4 expression in the elbow was similar to that of $\mathrm{R} 3+\mathrm{R} 4+\mathrm{R} 5$.

(TIF)

S5 Fig. Mutations in predicted PITX1 and ZEB1 binding sites do not alter R2d+e expression patterns. (A) Schematic representation of R2 sub-regions $\mathrm{R} 2 \mathrm{~d}$ and $\mathrm{R} 2 \mathrm{e}$, and the locations (asterisk, ${ }^{*}$ ) of two predicted transcription factor binding sites for PITX1 and ZEB1. A nineway species alignment reveals the high degree of sequence conservation in and around each predicted binding site (underlined). Red bases show the bases mutated to produce the $\Delta$ Pitx 1 and $\Delta$ Zeb1 enhancer constructs. (B) Comparison of transgenic embryos carrying the wild type $(\mathrm{R} 2 \mathrm{~d}+\mathrm{e})$, or mutant enhancer constructs ( $\Delta \mathrm{Pitx} 1$ and $\Delta \mathrm{Zeb} 1)$. All constructs drove similar lacZ expression in the proximal limb joints of shoulder (s), elbow (e), hip, and knee (k). Expression patterns outside the limb were not consistent for either the R2d+e construct or the constructs with mutant binding sites.

S6 Fig. Comparison of expression patterns of $G d f 5$ and neighboring genes Cep250 and Uqcc1. Panels show near adjacent sections of developing E15 hindlimbs (top row, A-D) and knees (bottom row, E-H), hybridized with probes for the indicated genes, or stained for Gdf5 Downstream BAC-LacZ expression. (A, E) Expression of Gdf5 in joints of the hindlimb and knee. (B, F) lacZ activity driven by the Downstream BAC shows expression in the same joint structures as the endogenous $G d f 5$ gene. In contrast, (C, G) the Cep250 gene shows weak expression in muscle tissue of the limb (red arrowheads), and (D, H) the Uqcc1 shows little or no concentrated staining in particular structures of the hindlimb at this stage. Abbreviations: ankle (a), femur (f), fibula (fi), and tibia (t).

(TIF)

S7 Fig. Osteoarthritis-associated risk alleles at 5'UTR positions do not alter qualitative patterns driven by human GDF5 constructs. (A) Adapted hg19 UCSC browser screenshot showing the 5 ' region of the human GDF5 locus, including the locations of two common human SNPs (rs143383 and rs143384) found in the 5'UTR. Derived alleles at these positions ("T, T") have previously been associated with increased risk of osteoarthritis [27]. (B) We generated two Hsp68 lacZ expression constructs containing an identical 1468 base pair region (hg19, chr20: 34,025,720-34,027,187 corresponding to +367 to $-1,101$ of the GDF5 promoter region [27]) that differed only by having ancestral ("C, C") or derived risk ("T, T") alleles at the rs143383 and rs143384 positions. Both constructs drove similar joint expression patterns in E14.5 day transgenic embryos. Abbreviations: shoulder (s), elbow (e), and knee (k). (TIF)

S8 Fig. Evolutionary conservation and chromatin mark labeling of regulatory sequences. UCSC Genome Browser view of a $130 \mathrm{~kb}$ region surrounding the human GDF5 locus on chromosome 20 (genome version hg19). This view highlights the genomic locations of the coding 
exons of GDF5 and UQCC (track: UCSC Genes); the position of five human sequences orthologous to the functionally defined mouse enhancers R1-R5 (track: GDF5 Regulatory Elements); and larger patterns of evolutionary sequence conservation based on 100 sequenced vertebrates (Tracks: 100 vertebrates Basewise Conservation by PhyloP; Multiz Alignments of 100 Vertebrates). Histone marks from chromatin immunoprecipitation (ChIP-seq) tracks in human and mouse tissues are shown as separate tracks. Data from Cotney et al (2013) [102] shows the locations H3K27ac peaks (active enhancers) in developing human and mouse limbs at four embryonic timepoints (E33, E41, E44, and E47) corresponding to equivalent mouse (mm9) gestational days (E10.5, E11.5, E12.5, and E13.5). Note that the downstream enhancer regions R3-R5 show peaks of H3k27ac signal at times of active joint formation in both humans and mice (Hg19E44+Hg19E47; Mm9E12.5+Mm9E13.5), but not at early stages when joints have not begun to form (Hg19E33+Hg19E41; Mm9E10.5+Mm9E11.5). A second experimental data track, acquired from the Roadmap Epigenomics Project (see text) [103], consists of significant $\mathrm{H} 3 \mathrm{~K} 27 \mathrm{ac}$ peaks in chondrocytes derived from adult human bone marrow. Note that H3K27ac peaks are seen over R1-R5 in human chondrocytes from four separate donors.

(TIF)

\section{Acknowledgments}

The authors would like to thank Catherine Guenther (Stanford University), Doug Menke (University of Georgia), and Aaron Wenger (Stanford University) for generous help and advice on constructs and transcription factor analyses, and members of the Kingsley and Capellini labs for useful discussions and comments on the manuscript.

\section{Author Contributions}

Conceptualization: HC TDC MS DPM DMK.

Formal analysis: HC TDC DMK.

Funding acquisition: DMK.

Investigation: HC TDC MS DPM AHR DPM.

Methodology: HC TDC MS DPM AHR DMK.

Project administration: DMK.

Resources: HC TDC DMK.

Supervision: DMK.

Validation: HC TDC.

Visualization: HC TDC.

Writing - original draft: HC TDC DMK.

Writing - review \& editing: HC TDC MS DPM AHR DMK.

\section{References}

1. Makin HJ. Struncture and functions of the joint. In Arthritis and Allied Conditions: A Textbook of Rheumatology. London: Williams and Wilkins Press; 1997.

2. Lawrence RC, Felson DT, Helmick CG, Arnold LM, Choi H, Deyo RA, et al. Estimates of the prevalence of arthritis and other rheumatic conditions in the United States. Part II. Arthritis Rheum. 2008; 58 (1):26-35. Epub 2008/01/01. doi: 10.1002/art.23176 PMID: 18163497 
3. Loughlin J. Genetic contribution to osteoarthritis development: current state of evidence. Curr Opin Rheumatol. 2015; 27(3):284-8. doi: 10.1097/BOR.0000000000000171 PMID: 25775188

4. Glyn-Jones S, Palmer AJ, Agricola R, Price AJ, Vincent TL, Weinans $\mathrm{H}$, et al. Osteoarthritis. Lancet. 2015; 386(9991):376-87. doi: 10.1016/S0140-6736(14)60802-3 PMID: 25748615

5. Urist MR. Bone morphogenetic protein: the molecularization of skeletal system development. J Bone Miner Res. 1997; 12(3):343-6. Epub 1997/03/01. doi: 10.1359/jbmr.1997.12.3.343 PMID: 9076576

6. Wozney JM, Rosen V. Bone morphogenetic protein and bone morphogenetic protein gene family in bone formation and repair. Clin Orthop Relat Res. 1998;(346: ):26-37. Epub 1998/05/13. PMID: 9577407

7. Storm EE, Huynh TV, Copeland NG, Jenkins NA, Kingsley DM, Lee SJ. Limb alterations in brachypodism mice due to mutations in a new member of the TGF beta-superfamily. Nature. 1994; 368 (6472):639-43. Epub 1994/04/14. doi: 10.1038/368639a0 PMID: 8145850

8. Chang SC, Hoang B, Thomas JT, Vukicevic S, Luyten FP, Ryba NJ, et al. Cartilage-derived morphogenetic proteins. New members of the transforming growth factor-beta superfamily predominantly expressed in long bones during human embryonic development. J Biol Chem. 1994; 269(45):2822734. Epub 1994/11/11. PMID: 7961761

9. Storm EE, Kingsley DM. Joint patterning defects caused by single and double mutations in members of the bone morphogenetic protein (BMP) family. Development. 1996; 122(12):3969-79. Epub 1996/ 12/01. PMID: 9012517

10. Brunet LJ, McMahon JA, McMahon AP, Harland RM. Noggin, cartilage morphogenesis, and joint formation in the mammalian skeleton. Science. 1998; 280(5368):1455-7. Epub 1998/06/20. PMID 9603738

11. Merino R, Macias D, Ganan $Y$, Economides AN, Wang X, Wu Q, et al. Expression and function of Gdf5 during digit skeletogenesis in the embryonic chick leg bud. Dev Biol. 1999; 206(1):33-45. Epub 1999/01/27. doi: 10.1006/dbio.1998.9129 PMID: 9918693

12. Hartmann C, Tabin CJ. Wnt-14 plays a pivotal role in inducing synovial joint formation in the developing appendicular skeleton. Cell. 2001; 104(3):341-51. Epub 2001/03/10. PMID: 11239392

13. Rountree RB, Schoor $M$, Chen $H$, Marks ME, Harley $V$, Mishina $Y$, et al. BMP receptor signaling is required for postnatal maintenance of articular cartilage. PLoS Biol. 2004; 2(11):e355. doi: 10.1371/ journal.pbio.0020355 PMID: 15492776

14. Koyama E, Shibukawa Y, Nagayama M, Sugito H, Young B, Yuasa T, et al. A distinct cohort of progenitor cells participates in synovial joint and articular cartilage formation during mouse limb skeletogenesis. Dev Biol. 2008; 316(1):62-73. Epub 2008/02/26. doi: 10.1016/j.ydbio.2008.01.012 PMID: 18295755

15. Gruneberg $\mathrm{H}$, Lee AJ. The anatomy and development of brachypodism in the mouse. J Embryol Exp Morphol. 1973; 30(1):119-41. Epub 1973/08/01. PMID: 4729943

16. Storm EE, Kingsley DM. GDF5 coordinates bone and joint formation during digit development. Dev Biol. 1999; 209(1):11-27. Epub 1999/04/20. doi: 10.1006/dbio.1999.9241 PMID: 10208739

17. Harada M, Takahara M, Zhe P, Otsuji M, luchi Y, Takagi M, et al. Developmental failure of the intraarticular ligaments in mice with absence of growth differentiation factor 5. Osteoarthr Cartilage. 2007; 15(4):468-74. Epub 2006/10/21.

18. Daans M, Luyten FP, Lories RJ. GDF5 deficiency in mice is associated with instability-driven joint damage, gait and subchondral bone changes. Ann Rheum Dis. 2011; 70(1):208-13. Epub 2010/09/ 02. doi: 10.1136/ard.2010.134619 PMID: 20805298

19. Settle SH Jr., Rountree RB, Sinha A, Thacker A, Higgins K, Kingsley DM. Multiple joint and skeletal patterning defects caused by single and double mutations in the mouse Gdf6 and Gdf5 genes. Dev Biol. 2003; 254(1):116-30. PMID: 12606286

20. Langer LO Jr., Cervenka J, Camargo M. A severe autosomal recessive acromesomelic dysplasia, the Hunter-Thompson type, and comparison with the Grebe type. Hum Genet. 1989; 81(4):323-8. Epub 1989/03/01. PMID: 2703235

21. Thomas JT, Lin K, Nandedkar M, Camargo M, Cervenka J, Luyten FP. A human chondrodysplasia due to a mutation in a TGF-beta superfamily member. Nat Genet. 1996; 12(3):315-7. Epub 1996/03/ 01. doi: 10.1038/ng0396-315 PMID: 8589725

22. Thomas JT, Kilpatrick MW, Lin K, Erlacher L, Lembessis $P$, Costa $T$, et al. Disruption of human limb morphogenesis by a dominant negative mutation in CDMP1. Nat Genet. 1997; 17(1):58-64. Epub 1997/09/01. doi: 10.1038/ng0997-58 PMID: 9288098

23. Seemann P, Schwappacher R, Kjaer KW, Krakow D, Lehmann K, Dawson K, et al. Activating and deactivating mutations in the receptor interaction site of GDF5 cause symphalangism or brachydactyly 
type A2. J Clin Invest. 2005; 115(9):2373-81. Epub 2005/08/30. doi: 10.1172/JCI25118 PMID: 16127465

24. Polinkovsky A, Robin NH, Thomas JT, Irons M, Lynn A, Goodman FR, et al. Mutations in CDMP1 cause autosomal dominant brachydactyly type C. Nat Genet. 1997; 17(1):18-9. Epub 1997/09/01. doi: 10.1038/ng0997-18 PMID: 9288091

25. Schwabe GC, Turkmen S, Leschik G, Palanduz S, Stover B, Goecke TO, et al. Brachydactyly type C caused by a homozygous missense mutation in the prodomain of CDMP1. Am J Med Genet A. 2004; 124A(4):356-63. Epub 2004/01/22. doi: 10.1002/ajmg.a.20349 PMID: 14735582

26. Holder-Espinasse M, Escande F, Mayrargue E, Dieux-Coeslier A, Fron D, Doual-Bisser A, et al. Angel shaped phalangeal dysplasia, hip dysplasia, and positional teeth abnormalities are part of the brachydactyly C spectrum associated with CDMP-1 mutations. J Med Genet. 2004; 41(6):e78. Epub 2004/ 06/03. doi: 10.1136/jmg.2003.013904 PMID: 15173244

27. Miyamoto Y, Mabuchi A, Shi D, Kubo T, Takatori Y, Saito S, et al. A functional polymorphism in the 5' UTR of GDF5 is associated with susceptibility to osteoarthritis. Nat Genet. 2007; 39(4):529-33. Epub 2007/03/27. doi: 10.1038/2005 PMID: 17384641

28. Sanna S, Jackson AU, Nagaraja R, Willer CJ, Chen WM, Bonnycastle LL, et al. Common variants in the GDF5-UQCC region are associated with variation in human height. Nat Genet. 2008; 40(2):198203. Epub 2008/01/15. doi: 10.1038/ng.74 PMID: 18193045

29. Southam L, Rodriguez-Lopez J, Wilkins JM, Pombo-Suarez M, Snelling S, Gomez-Reino JJ, et al. An SNP in the 5'-UTR of GDF5 is associated with osteoarthritis susceptibility in Europeans and with in vivo differences in allelic expression in articular cartilage. Hum Mol Genet. 2007; 16(18):2226-32. Epub 2007/07/10. doi: 10.1093/hmg/ddm174 PMID: 17616513

30. Egli RJ, Southam L, Wilkins JM, Lorenzen I, Pombo-Suarez M, Gonzalez A, et al. Functional analysis of the osteoarthritis susceptibility-associated GDF5 regulatory polymorphism. Arthritis Rheum. 2009; 60(7):2055-64. Epub 2009/07/01. doi: 10.1002/art.24616 PMID: 19565498

31. Dodd AW, Rodriguez-Fontenla C, Calaza M, Carr A, Gomez-Reino JJ, Tsezou A, et al. Deep sequencing of GDF5 reveals the absence of rare variants at this important osteoarthritis susceptibility locus. Osteoarthr Cartilage. 2011; 19(4):430-4. Epub 2011/02/02.

32. Wu DD, Li GM, Jin W, Li Y, Zhang YP. Positive Selection on the Osteoarthritis-Risk and DecreasedHeight Associated Variants at the GDF5 Gene in East Asians. PLoS One. 2012; 7(8):e42553. Epub 2012/08/21. doi: 10.1371/journal.pone.0042553 PMID: 22905146

33. Dodd AW, Syddall CM, Loughlin J. A rare variant in the osteoarthritis-associated locus GDF5 is functional and reveals a site that can be manipulated to modulate GDF5 expression. Eur J Hum Genet. 2013; 21(5):517-21. Epub 2012/08/30. doi: 10.1038/ejhg.2012.197 PMID: 22929025

34. Bobacz K, Sunk IG, Hayer S, Amoyo L, Tohidast-Akrad M, Kollias G, et al. Differentially regulated expression of growth differentiation factor 5 and bone morphogenetic protein 7 in articular cartilage and synovium in murine chronic arthritis: potential importance for cartilage breakdown and synovial hypertrophy. Arthritis Rheum. 2008; 58(1):109-18. doi: 10.1002/art.23145 PMID: 18163510

35. Kan A, Ikeda T, Fukai A, Nakagawa T, Nakamura K, Chung UI, et al. SOX11 contributes to the regulation of GDF5 in joint maintenance. BMC Dev Biol. 2013; 13:4. doi: 10.1186/1471-213X-13-4 PMID: 23356643

36. Newburger DE, Bulyk ML. UniPROBE: an online database of protein binding microarray data on protein-DNA interactions. Nucleic Acids Res. 2009; 37(Database issue):D77-82. Epub 2008/10/10. doi: 10.1093/nar/gkn660 PMID: 18842628

37. Quandt K, Frech K, Karas H, Wingender E, Werner T. MatInd and MatInspector: new fast and versatile tools for detection of consensus matches in nucleotide sequence data. Nucleic Acids Res. 1995; 23 (23):4878-84. Epub 1995/12/11. PMID: 8532532

38. Loots GG, Ovcharenko I, Pachter L, Dubchak I, Rubin EM. rVista for comparative sequence-based discovery of functional transcription factor binding sites. Genome Res. 2002; 12(5):832-9. Epub 2002/ 05/09. 10.1101/gr.225502. Article published online before print in April 2002. doi: 10.1101/gr.225502 PMID: 11997350

39. Loots GG, Ovcharenko I. rVISTA 2.0: evolutionary analysis of transcription factor binding sites. Nucleic Acids Res. 2004; 32(Web Server issue):W217-21. doi: 10.1093/nar/gkh383 PMID: 15215384

40. Cartharius K, Frech K, Grote K, Klocke B, Haltmeier M, Klingenhoff A, et al. MatInspector and beyond: promoter analysis based on transcription factor binding sites. Bioinformatics. 2005; 21(13):2933-42. Epub 2005/04/30. doi: 10.1093/bioinformatics/bti473 PMID: 15860560

41. Meech R, Edelman DB, Jones FS, Makarenkova HP. The homeobox transcription factor Barx2 regulates chondrogenesis during limb development. Development. 2005; 132(9):2135-46. doi: 10.1242/ dev.01811 PMID: 15800003 
42. Smith DM, Tabin CJ. Chick Barx2b, a marker for myogenic cells also expressed in branchial arches and neural structures. Mech Develop. 1999; 80(2):203-6.

43. Barlow AJ, Bogardi JP, Ladher R, Francis-West PH. Expression of chick Barx-1 and its differential regulation by FGF-8 and BMP signaling in the maxillary primordia. Dev Dynam. 1999; 214(4):291-302.

44. Gray PA, Fu H, Luo $P, Z$ hao $Q$, Yu J, Ferrari A, et al. Mouse brain organization revealed through direct genome-scale TF expression analysis. Science. 2004; 306(5705):2255-7. doi: 10.1126/science. 1104935 PMID: 15618518

45. Ingham PW. The molecular genetics of embryonic pattern formation in Drosophila. Nature. 1988; 335 (6185):25-34. doi: 10.1038/335025a0 PMID: 2901040

46. Howard KR, Struhl G. Decoding positional information: regulation of the pair-rule gene hairy. Development. 1990; 110(4):1223-31. PMID: 2100260

47. Small S, Levine M. The initiation of pair-rule stripes in the Drosophila blastoderm. Curr Opin Genet Dev. 1991; 1(2):255-60. PMID: 1822273

48. Stanojevic $D$, Small S, Levine M. Regulation of a segmentation stripe by overlapping activators and repressors in the Drosophila embryo. Science. 1991; 254(5036):1385-7. PMID: 1683715

49. Jaynes JB, Fujioka M. Drawing lines in the sand: even skipped et al. and parasegment boundaries. Dev Biol. 2004; 269(2):609-22. doi: 10.1016/j.ydbio.2004.03.001 PMID: 15110723

50. Hume MA, Barrera LA, Gisselbrecht SS, Bulyk ML. UniPROBE, update 2015: new tools and content for the online database of protein-binding microarray data on protein-DNA interactions. Nucleic Acids Res. 2015; 43(Database issue):D117-22. doi: 10.1093/nar/gku1045 PMID: 25378322

51. Gibson-Brown JJ, Agulnik SI, Chapman DL, Alexiou M, Garvey N, Silver LM, et al. Evidence of a role for T-box genes in the evolution of limb morphogenesis and the specification of forelimb/hindlimb identity. Mech Develop. 1996; 56(1-2):93-101.

52. Shang J, Luo Y, Clayton DA. Backfoot is a novel homeobox gene expressed in the mesenchyme of developing hind limb. Develop Dynam. 1997; 209(2):242-53.

53. Takagi $\mathrm{T}$, Moribe $\mathrm{H}$, Kondoh $\mathrm{H}$, Higashi $\mathrm{Y}$. DeltaEF1, a zinc finger and homeodomain transcription factor, is required for skeleton patterning in multiple lineages. Development. 1998; 125(1):21-31. PMID: 9389660

54. Szeto DP, Rodriguez-Esteban C, Ryan AK, O'Connell SM, Liu F, Kioussi C, et al. Role of the Bicoidrelated homeodomain factor Pitx1 in specifying hindlimb morphogenesis and pituitary development. Gene Dev. 1999; 13(4):484-94. PMID: 10049363

55. Lanctot $C$, Moreau A, Chamberland M, Tremblay ML, Drouin J. Hindlimb patterning and mandible development require the Ptx1 gene. Development. 1999; 126(9):1805-10. PMID: 10101115

56. Margulies EH, Kardia SL, Innis JW. A comparative molecular analysis of developing mouse forelimbs and hindlimbs using serial analysis of gene expression (SAGE). Genome Res. 2001; 11(10):1686-98. doi: 10.1101/gr.192601 PMID: 11591645

57. Ahn DG, Kourakis MJ, Rohde LA, Silver LM, Ho RK. T-box gene tbx5 is essential for formation of the pectoral limb bud. Nature. 2002; 417(6890):754-8. doi: 10.1038/nature00814 PMID: 12066188

58. Naiche LA, Papaioannou VE. Loss of Tbx4 blocks hindlimb development and affects vascularization and fusion of the allantois. Development. 2003; 130(12):2681-93. PMID: 12736212

59. Ferrari $D$, Kosher RA. Expression of DIx 5 and Dlx6 during specification of the elbow joint. Int J Dev Biol. 2006; 50(8):709-13. doi: 10.1387/ijdb.062180df PMID: 17051482

60. DeLaurier A, Schweitzer R, Logan M. Pitx1 determines the morphology of muscle, tendon, and bones of the hindlimb. Dev Biol. 2006; 299(1):22-34. doi: 10.1016/j.ydbio.2006.06.055 PMID: 16989801

61. Spater D, Hill TP, Gruber M, Hartmann C. Role of canonical Wnt-signalling in joint formation. Eur Cells Mater. 2006; 12:71-80.

62. Dy P, Smits $\mathrm{P}$, Silvester A, Penzo-Mendez A, Dumitriu B, Han Y, et al. Synovial joint morphogenesis requires the chondrogenic action of Sox 5 and Sox6 in growth plate and articular cartilage. Dev Biol. 2010; 341(2):346-59. doi: 10.1016/j.ydbio.2010.02.024 PMID: 20206616

63. Villavicencio-Lorini $\mathrm{P}$, Kuss $\mathrm{P}$, Friedrich J, Haupt J, Farooq M, Turkmen S, et al. Homeobox genes d11-d13 and a13 control mouse autopod cortical bone and joint formation. J Clin Invest. 2010; 120 (6):1994-2004. doi: 10.1172/JCI41554 PMID: 20458143

64. Pazin DE, Gamer LW, Cox KA, Rosen V. Molecular profiling of synovial joints: use of microarray analysis to identify factors that direct the development of the knee and elbow. Develop Dynam. 2012; 241 (11):1816-26. Epub 2012/09/14.

65. Kan A, Tabin CJ. c-Jun is required for the specification of joint cell fates. Gene Dev. 2013; 27(5):51424. doi: 10.1101/gad.209239.112 PMID: 23475960 
66. Tissier-Seta JP, Mucchielli ML, Mark M, Mattei MG, Goridis C, Brunet JF. Barx1, a new mouse homeodomain transcription factor expressed in cranio-facial ectomesenchyme and the stomach. Mech Develop. 1995; 51(1):3-15.

67. Church V, Yamaguchi K, Tsang $P$, Akita $K$, Logan $C$, Francis-West $P$. Expression and function of Bapx1 during chick limb development. Anat Embryol. 2005; 209(6):461-9. doi: 10.1007/s00429-0050464-z PMID: 15887045

68. Crotwell PL, Clark TG, Mabee PM. Gdf5 is expressed in the developing skeleton of median fins of latestage zebrafish, Danio rerio. Dev Genes Evol. 2001; 211(11):555-8. doi: 10.1007/s00427-001-0186-z PMID: 11862461

69. Mitchell RE, Huitema LF, Skinner RE, Brunt LH, Severn C, Schulte-Merker S, et al. New tools for studying osteoarthritis genetics in zebrafish. Osteoarthr Cartilage. 2013; 21(2):269-78.

70. Searcy RD, Vincent EB, Liberatore CM, Yutzey KE. A GATA-dependent nkx-2.5 regulatory element activates early cardiac gene expression in transgenic mice. Development. 1998; 125(22):4461-70. PMID: 9778505

71. Lien CL, Wu C, Mercer B, Webb R, Richardson JA, Olson EN. Control of early cardiac-specific transcription of Nkx2-5 by a GATA-dependent enhancer. Development. 1999; 126(1):75-84. PMID: 9834187

72. Reecy JM, Li X, Yamada M, DeMayo FJ, Newman CS, Harvey RP, et al. Identification of upstream regulatory regions in the heart-expressed homeobox gene Nkx2-5. Development. 1999; 126(4):83949. PMID: 9895330

73. Tanaka M, Wechsler SB, Lee IW, Yamasaki N, Lawitts JA, Izumo S. Complex modular cis-acting elements regulate expression of the cardiac specifying homeobox gene Csx/Nkx2.5. Development. 1999; 126(7):1439-50. PMID: 10068637

74. Schwartz RJ, Olson EN. Building the heart piece by piece: modularity of cis-elements regulating Nkx25 transcription. Development. 1999; 126(19):4187-92. PMID: 10477287

75. Mortlock DP, Guenther C, Kingsley DM. A general approach for identifying distant regulatory elements applied to the Gdf6 gene. Genome Res. 2003; 13(9):2069-81. Epub 2003/08/14. doi: 10.1101/gr. 1306003 PMID: 12915490

76. Guenther CA, Wang Z, Li E, Tran MC, Logan CY, Nusse R, et al. A distinct regulatory region of the Bmp5 locus activates gene expression following adult bone fracture or soft tissue injury. Bone. 2015 77:31-41. doi: 10.1016/j.bone.2015.04.010 PMID: 25886903

77. Indjeian VB, Kingman GA, Jones FC, Guenther CA, Grimwood J, Schmutz J, et al. Evolving new skeletal traits by cis-regulatory changes in Bone Morphogenetic Proteins. Cell. 2016; 164(1-2):45-56. doi: 10.1016/j.cell.2015.12.007 PMID: 26774823

78. Summerbell D, Ashby PR, Coutelle O, Cox D, Yee S, Rigby PW. The expression of Myf5 in the developing mouse embryo is controlled by discrete and dispersed enhancers specific for particular populations of skeletal muscle precursors. Development. 2000; 127(17):3745-57. PMID: 10934019

79. Zammit PS, Carvajal JJ, Golding JP, Morgan JE, Summerbell D, Zolnerciks J, et al. Myf5 expression in satellite cells and spindles in adult muscle is controlled by separate genetic elements. Dev Biol. 2004; 273(2):454-65. doi: 10.1016/j.ydbio.2004.05.038 PMID: 15328025

80. Kingsley DM. What do BMPs do in mammals? Clues from the mouse short-ear mutation. Trends Genet. 1994; 10(1):16-21. PMID: 8146910

81. Wray GA. The evolutionary significance of cis-regulatory mutations. Nat Rev Genet. 2007; 8(3):20616. doi: $10.1038 / n r g 2063$ PMID: 17304246

82. Carroll SB. Evo-devo and an expanding evolutionary synthesis: a genetic theory of morphological evoIution. Cell. 2008; 134(1):25-36. doi: 10.1016/j.cell.2008.06.030 PMID: 18614008

83. Stern DL, Orgogozo V. Is genetic evolution predictable? Science. 2009; 323(5915):746-51. doi: 10. 1126/science.1158997 PMID: 19197055

84. Dawson K, Seeman P, Sebald E, King L, Edwards M, Williams J 3rd, et al. GDF5 is a second locus for multiple-synostosis syndrome. Am J Hum Genet. 2006; 78(4):708-12. doi: 10.1086/503204 PMID: 16532400

85. Seemann $\mathrm{P}$, Brehm A, Konig J, Reissner $\mathrm{C}$, Stricker $\mathrm{S}$, Kuss $\mathrm{P}$, et al. Mutations in GDF5 reveal a key residue mediating BMP inhibition by NOGGIN. PLoS Genet. 2009; 5(11):e1000747. doi: 10.1371/ journal.pgen.1000747 PMID: 19956691

86. Chapman K, Takahashi A, Meulenbelt I, Watson C, Rodriguez-Lopez J, Egli R, et al. A meta-analysis of European and Asian cohorts reveals a global role of a functional SNP in the 5' UTR of GDF5 with osteoarthritis susceptibility. Hum Mol Genet. 2008; 17(10):1497-504. Epub 2008/02/27. doi: 10.1093/ hmg/ddn038 PMID: 18299287 
87. Evangelou E, Chapman K, Meulenbelt I, Karassa FB, Loughlin J, Carr A, et al. Large-scale analysis of association between GDF5 and FRZB variants and osteoarthritis of the hip, knee, and hand. Arthritis Rheum. 2009; 60(6):1710-21. Epub 2009/05/30. doi: 10.1002/art.24524 PMID: 19479880

88. Vaes RB, Rivadeneira F, Kerkhof JM, Hofman A, Pols HA, Uitterlinden AG, et al. Genetic variation in the GDF5 region is associated with osteoarthritis, height, hip axis length and fracture risk: the Rotterdam study. Ann Rheum Dis. 2009; 68(11):1754-60. Epub 2008/11/26. doi: 10.1136/ard.2008.099655 PMID: 19029166

89. Valdes AM, Spector TD, Doherty S, Wheeler M, Hart DJ, Doherty M. Association of the DVWA and GDF5 polymorphisms with osteoarthritis in UK populations. Ann Rheum Dis. 2009; 68(12):1916-20. Epub 2008/12/05. doi: 10.1136/ard.2008.102236 PMID: 19054821

90. Ji J, Dai J, Shi D, Jiang Q. Association of genetic and mechanical factors with age of onset of knee osteoarthritis. Chin J Med Genet. 2010; 27(6):672-4.

91. Valdes AM, Evangelou E, Kerkhof HJ, Tamm A, Doherty SA, Kisand K, et al. The GDF5 rs143383 polymorphism is associated with osteoarthritis of the knee with genome-wide statistical significance. Ann Rheum Dis. 2011; 70(5):873-5. Epub 2010/09/28. doi: 10.1136/ard.2010.134155 PMID: 20870806

92. Liu J, Cai W, Zhang H, He C, Deng L. Rs143383 in the growth differentiation factor 5 (GDF5) gene significantly associated with osteoarthritis (OA) - a comprehensive meta-analysis. Int J Med Sci. 2013; 10(3):312-9. doi: 10.7150/ijms.5455 PMID: 23423687

93. Lee EC, Yu D, Martinez de Velasco J, Tessarollo L, Swing DA, Court DL, et al. A highly efficient Escherichia coli-based chromosome engineering system adapted for recombinogenic targeting and subcloning of BAC DNA. Genomics. 2001; 73(1):56-65. doi: 10.1006/geno.2000.6451 PMID: 11352566

94. DiLeone RJ, Russell LB, Kingsley DM. An extensive 3' regulatory region controls expression of Bmp5 in specific anatomical structures of the mouse embryo. Genetics. 1998; 148(1):401-8. PMID: 9475750

95. DiLeone RJ, Marcus GA, Johnson MD, Kingsley DM. Efficient studies of long-distance Bmp5 gene regulation using bacterial artificial chromosomes. Proc Natl Acad Sci U S A. 2000; 97(4):1612-7. PMID: 10677507

96. Wilkinson DG. Whole mount in situ hybridization of vertebrate embryos. In In Situ Hybridization: A Practical Approach. Oxford: IRL Press; 1992.

97. Lufkin T, Mark M, Hart CP, Dolle P, LeMeur M, Chambon P. Homeotic transformation of the occipital bones of the skull by ectopic expression of a homeobox gene. Nature. 1992; 359(6398):835-41. doi: 10.1038/359835a0 PMID: 1359423

98. Mayor C, Brudno M, Schwartz JR, Poliakov A, Rubin EM, Frazer KA, et al. VISTA: visualizing global DNA sequence alignments of arbitrary length. Bioinformatics. 2000; 16(11):1046-7. PMID: 11159318

99. Brudno M, Do CB, Cooper GM, Kim MF, Davydov E, Program NCS, et al. LAGAN and Multi-LAGAN: efficient tools for large-scale multiple alignment of genomic DNA. Genome Res. 2003; 13(4):721-31. doi: 10.1101/gr.926603 PMID: 12654723

100. Berger MF, Bulyk ML. Protein binding microarrays (PBMs) for rapid, high-throughput characterization of the sequence specificities of DNA binding proteins. Methods Mol Biol. 2006; 338:245-60. Epub 2006/08/05. doi: 10.1385/1-59745-097-9:245 PMID: 16888363

101. Ho SN, Hunt HD, Horton RM, Pullen JK, Pease LR. Site-directed mutagenesis by overlap extension using the polymerase chain reaction. Gene. 1989; 77(1):51-9. PMID: 2744487

102. Cotney J, Leng J, Yin J, Reilly SK, DeMare LE, Emera D, et al. The evolution of lineage-specific regulatory activities in the human embryonic limb. Cell. 2013; 154(1):185-96. doi: 10.1016/j.cell.2013.05. 056 PMID: 23827682

103. Roadmap Epigenomics C, Kundaje A, Meuleman W, Ernst J, Bilenky M, Yen A, et al. Integrative analysis of 111 reference human epigenomes. Nature. 2015; 518(7539):317-30. doi: 10.1038/ nature14248 PMID: 25693563 